\title{
Loss of TCF-1 regulates production of noncanonical Tregs in a prion-like manner
}

\author{
Rebecca Harris, Mahinbanu Mammadli, Mobin Karimi \\ Dept. of Microbiology and Immunology, SUNY Upstate Medical University, Syracuse, NY \\ 13210
}

Summary sentence: Loss of TCF-1 causes expansion of CD25- FOXP3+ noncanonical Tregs, and TCF-1-deficient T cells induce increased production of CD25- Tregs from WT cells.

Running Title: TCF-1 controls CD25- Treg expansion in mice

To whom correspondence should be addressed:

Mobin Karimi

Assistant Professor of Immunology and Microbiology

SUNY Upstate Medical University,

766 Irving Ave Weiskotten Hall Suite 2281,

Syracuse, NY 13210

Office Phone: 315-464-2344

Laboratory Phone: 315-464-7652 Email: karimim@upstate.edu

Key Words: T cells, FOXP3, suppressive 


\section{ABBREVIATIONS}

2 cGys - centiGrays

$3 \mathrm{cKO}$ - conditional knock-out

4 Eomes - Eomesodermin

5 IPEX - immune dysregulation, polyendocrinopathy, and X-linked syndrome

$6 \mathrm{KO}-$ knock-out

7 LAK media- lymphocyte activated killer media

$8 \mathrm{MHC}(\mathrm{I} / \mathrm{II})$ - major histocompatibility complex (I/II)

9 RBCs - red blood cells

10 T-bet - T-box transcription factor TBX21

11 TCF-1/TCF - T Cell Factor-1

12 Tregs - regulatory T cells

13 WT - wild-type 


\section{$24 \underline{\text { Abstract }}$}

25 Regulatory T cells (Tregs) are suppressive immune cells used for a variety of clinical and

26 therapeutic applications. Canonical Tregs express CD4, FOXP3, and CD25, which are

27 considered definitive markers of Treg status when used together. However, a subset of

28 noncanonical Tregs expressing only CD4 and FOXP3 have recently been described in some

29 infection contexts. The transcriptional regulation of these cells is still unclear. We found that loss

30 of TCF-1 in all T cells in mice leads to expansion of these cells in multiple tissues in a cell-

31 intrinsic fashion. This effect was not due to aberrant expression of FOXP3, as other functional

32 Treg markers were also expressed. In addition, presence of TCF-1-deficient cells in a chimeric

33 mouse induced increased production of noncanonical Tregs from WT donor cells. Therefore,

34 targeting of TCF-1 may remove suppression on this Treg lineage, increasing the yield of these

35 cells for use in the clinic. 


\section{$\underline{\text { Introduction }}$}

T cells are critical for production of an immune response against many types of threats.

$47 \mathrm{CD} 8+\mathrm{T}$ cells are cytotoxic cells that recognize specific peptide/MHC I complexes, whereas

$48 \mathrm{CD} 4+\mathrm{T}$ cells recognize specific peptide/MHC II complexes and provide help to developing

49 immune cells ${ }^{1}$. Overactivation of T cells may lead to a state of chronic inflammation and

50 immunopathology, so several mechanisms are in place to avoid inappropriate activation of $\mathrm{T}$

51 cells. The main mechanism that governs appropriate $\mathrm{T}$ cell reactivity is thymic education, which

52 involves positive selection and negative selection of $\mathrm{T}$ cells ${ }^{2}$. This process generates $\mathrm{T}$ cells that

53 react weakly to self MHC/peptide complexes (positive selection) but deletes T cells that

54 recognize self $\mathrm{MHC} /$ peptide complexes too strongly (negative selection) $)^{2,3,4}$. However, a small

55 fraction of T cells may avoid negative selection, which could potentially lead to autoimmunity.

56 When this occurs, peripheral mechanisms act to prevent the activation of these potentially

57 autoreactive $\mathrm{T}$ cells $\mathrm{s}^{2,5}$. One such mechanism involves the suppression of $\mathrm{T}$ cell activation by

58 regulatory $\mathrm{T}$ cells (Tregs), a subset of T cells with inhibitory function ${ }^{6}$. The importance of Tregs

59 in protection against autoimmunity is exemplified by the systemic fatal autoimmunity that

60 develops in Scurfy mice ${ }^{7,8}$, which are deficient in Tregs, as well as in patients with IPEX

61 (immune dysregulation, polyendocrinopathy, and X-linked) syndrome ${ }^{9}$. Scurfy mice and IPEX

62 syndrome patients harbor a mutation in FoxP3 $3^{7,8,9}$, which is essential for Treg development and

63 function ${ }^{10}$. Thus, the presence of FoxP3+ Tregs is critical for the induction of peripheral

64 tolerance.

65

66 Canonical Tregs are identified by expression of FOXP3 along with CD25, but reports

67 have identified a noncanonical population of Tregs which is FOXP3 + CD25- ${ }^{11,12}$. Unlike 
68 developing cells in the thymus, which can also express FOXP3 but not CD25, these

69 noncanonical Tregs are found in the periphery lacking expression of CD25. These noncanonical

70 Tregs are suppressive in several experimental models, including autoimmune encephalitis,

71 inflammatory bowel disease, allergy, and diabetes models ${ }^{13,14,15,16,17}$. Despite the observation of

72 these cells in many context, it is still unknown exactly which transcription factors drive

73 production of these Tregs. However, CD25-deficient mice are unable to produce suppressive

74 Tregs, suggesting that CD25 expression is required at some point for suppressive ability of

75 CD25- Tregs, and is later lost in these cells ${ }^{13}$.

Tregs help to maintain tolerance and immune homeostasis by inhibiting the proliferation

78 of and cytokine production by other $\mathrm{T}$ cells ${ }^{6,10,18}$. It is uncertain exactly how Tregs suppress other

79 cells, but a variety of mechanisms have been described. These include: cell-contact dependent

80 suppression (such as through Treg-expressed CTLA-4 binding to CD28 on the target cell),

81 release of inhibitory cytokines (i.e. IL-10 and TGF-b), adsorption of IL-2, or modulating

82 dendritic cell co-stimulation ${ }^{18}$. Using quantitative modeling, a recent study has also suggested

83 that the major mechanism of Treg suppression is through a reduction in division destiny - the

84 number of total divisions before a cell becomes quiescent - rather than reduced proliferation or

85 increased cell death ${ }^{18}$. Although the functional mechanisms of these cells are still unclear, Tregs

86 are known to express CTLA-4, IL-10, and TGF-b when they are functionally capable of

87 suppression $^{19,20,21,22}$. 
T Cell Factor-1 (TCF-1) was recently shown to play a role in Treg survival when in 90 combination with LEF-1 $1^{23}$. TCF-1 has also recently been identified as a suppressive factor of FOXP3, as it binds to the promoter region of FOXP3 to prevent aberrant expression of FOXP3 in

92

conventional T cells ${ }^{24}$. Delacher et al. [2020] used viral overexpression of TCF-1 in T cells to show that TCF-1 suppresses induction of FOXP3 in conventional T cells under Treg-inducing culture conditions. In addition, they showed that mice with global TCF-1 deficiency had an increased frequency of CD25- FOXP $3^{\text {int }} \mathrm{T}$ cells, and that FOXP3 expression was also increased among CD8 T cells. These data, combined with the finding that these cells did not express CTLA-4 as expected, led the authors to suggest that loss of TCF-1 leads to aberrant FOXP3 expression in T cells, not to expansion of true Tregs. Finally, the authors used CRISPR to knock out TCF-1 in human and mouse CD4 T cells, and found an increase in FOXP3+ CD4 T cells ${ }^{24}$.

However, TCF-1 is a critical T cell transcription factor for T cell development, CD4/CD8

lineage maintenance, and responses to infection $25,26,27,28,29,30,31,32$, so global deficiency of this factor drastically influences T cell development and function ${ }^{33}$. It is unknown whether TCF-1 deficiency in mature $\mathrm{T}$ cells has a similar effect on FOXP3. The potential for off-target effects of CRISPR systems is also well-documented ${ }^{34,35,36}$, leading to potentially faulty conclusions on the true role of TCF-1 in controlling mature T cell expression of FOXP3. Our studies instead utilized a T cell-specific deletion of TCF-1 ${ }^{37}$ to investigate the effects of TCF-1 loss in mature cells on canonical (CD25+ FOXP3+) and noncanonical (CD25-FOXP3+) Tregs. We found that loss of TCF-1 in mature T cells led to an increased in frequency and number of noncanonical, FOXP3+CD25- Tregs, with no impact on to frequency but a drop in numbers of canonical 
111 CD25+ Tregs. This effect was cell-intrinsic, and unaffected by changes to Eomes and T-bet,

112 which are altered when TCF-1 is lost. Eomesodermin (Eomes) and T-box transcription factor

113 TBX21 (T-bet) are downstream of TCF-1, and also play critical roles in maintaining T cell

114 lineage $\mathrm{e}^{32,38,39}$.

115

116

Of critical importance, when WT cells were present in the same microenvironment as

117 TCF-1-deficient Tregs (in a chimeric mouse), TCF cKO T cells induced elevated production of

118 noncanonical Tregs from WT donor cells. Therefore, TCF cKO T cells have a prion-like ability

119 to promote noncanonical Treg fate among nearby cells, despite the WT phenotype of these

120 neighboring cells. These CD25- Tregs were found in multiple tissues, and were not expanded

121 due to aberrant expression of FOXP3, because other functional Treg markers (CTLA-4 and IL-

122 10) were also expressed in these cells. IL-2 production was maintained in TCF cKO CD25-

123 Tregs at similar levels to WT CD25- and CD25+ Tregs. CD8 T cells expressing FOXP3 without

124 CD25 were not increased by loss of TCF-1. Thus, our results show that while TCF-1 does appear

125 to control FOXP3 expression, loss of TCF-1 in mature T cells does not simply cause FOXP3 to

126 be expressed aberrantly. Instead, TCF-1 deficiency induces expansion of a unique CD25-

127 FOXP3 + subset of Tregs which has recently been described in multiple models. Our observation

128 of a prion-like trait of TCF-1-deficient T cells to induce this phenotype in WT cells within the

129 same microenvironment is also novel. These studies show that TCF-1 modulation could be used

130 to affect noncanonical Treg production and yield, and may lead to a prion-like ability to affect

131 the fate of other nearby cells in the microenvironment. 


\section{Materials and Methods}

134 Mice. Thy1.1 (B6.PL-Thy1a/CyJ, 000406), B6-Ly5 (CD45.1+, AKA “WT” or B6.SJL-Ptprc

135 Pepc $c^{\mathrm{b}} /$ BoyJ, 002014), and BALB/c mice (CR:028) were purchased from Charles River or

136 Jackson Laboratory. TCF cKO mice (Tcf7 flox/flox x CD4cre) ${ }^{37}$ were obtained from Dr. Jyoti

137 Misra Sen at the NIH and bred in our facilities. CD4cre (022071), Eomes flox/flox (017293), and

138 T-bet flox/flox (022741) mice were purchased from Jackson Laboratories. CD4cre mice were

139 bred in our facilities with Eomes or T-bet flox mice to produce Eomes cKO or T-bet cKO mice,

140 respectively. All mice used for transplants were female, and flow cytometry experiments were

141 done with both male and female mice. All animal experiments were approved by the IACUC at

142 SUNY Upstate Medical University. All procedures (including animal maintenance) were

143 performed according to the rules and guidance provided by the IACUC. Mice aged 8-12 weeks

144 were used, and all experiments were performed with age and sex-matched mice.

145 Flow cytometry. To analyze expression of Treg markers, lymphocytes were collected and stained

146 for flow cytometry. Lymphocytes were obtained from organs, filtered with a 70uM filter, and

147 treated with RBC Lysis Buffer to remove red blood cells (RBCs). The cells were then washed

148 with ice-cold MACS buffer (1x PBS with EDTA and 4g/L BSA) and plated in a 96-well V-

149 bottom plate. Antibody cocktails were prepared in 1x PBS or MACS buffer and added to each

150 well. The cells were stained for 30 minutes on ice, covered to protect from light. The cells were

151 then spun to remove antibodies and washed 1-2 times with ice-cold 1x PBS or MACS. Cells

152 were fixed overnight at $4 \mathrm{C}$ in $200 \mathrm{uL}$ of fixative (Fix/Perm Concentrate and Fixation Diluent

153 from FOXP3 Transcription Factor Staining Buffer Set, eBioscience cat. No. 00-5523-00). The

154 next day, stained cells were permeabilized by washing twice with permeabilization buffer 
155

156

(eBioscience cat. No. 00-5523-00) and then stained for intracellular markers for 40min at room temperature with antibody in perm buffer, covered from light. Cells were then washed 1-2 times

157 with perm buffer, resuspended in 200-400uL of FACS buffer (eBioscience cat. No. 00-4222-26),

158

159 and transferred to flow tubes. Data was collected on a BD LSRFortessa cytometer (BD Biosciences). Data were analyzed using FlowJo v9 (Treestar).

160

Antibodies. All antibodies were purchased from eBiosciences, Biolegend, or BD Biosciences.

161 Antibodies used included: anti-CD4-BV785, anti-FOXP3-APC, anti-CD25-PE, anti-CD25BV421, anti-CD45.2-PE/Cy7, anti-CD45.1-Pacific Blue, anti-Thy1.1-AF700, anti-Thy1.2-APC, 163 anti-CD4-FITC, anti-CD45.1-PE, anti-CD3-APC/Cy7, anti-CD8-FITC, anti-CD8-PE, anti-IL-2PE/Cy7, anti-CTLA-4-PE, and anti-IL-10-APC/Cy7. LIVE/DEAD Fixable Aqua Dead Cell Stain 165 (Invitrogen cat. No. L34957) was used to remove dead cells from the analysis. Anti-CD3 (clone 17A2, Biolegend cat. no. 100202) was used to coat stimulation plates, and Ultra LEAF-purified anti-CD28 (clone 37.51, Biolegend cat. no. 102116) was used as a soluble stimulator.

Chimera Production. To produce bone marrow chimeras, Thy1.1 female mice aged 8-12 weeks were lethally irradiated with $800 \mathrm{cGys}$ in a single dose. Bone marrow was isolated from femur and tibia of WT (B6Ly5, CD45.1) and TCF cKO (CD45.2) mice, filtered through a 70uM filter,

171 and counted. Bone marrow cells were mixed at a 1:4 (WT:TCF cKO) ratio to ensure survival of $172 \mathrm{KO}$ cells with a potential proliferation defect. Bone marrow cells were suspended in sterile $1 \mathrm{x}$ 173 PBS for transplantation. The mixed bone marrow was injected into the Thy 1.1 recipients via the 174 tail vein at 4 hours post-irradiation. At 9 weeks post-transplant, blood was collected from the 175 Thy1.1 mice and tested via flow cytometry for presence of both CD45.1 (WT) and CD45.2 (TCF 
176

177

178

179 euthanized and the livers were perfused with $5 \mathrm{~mL}$ of cold 1x PBS. The livers were then mashed

180

181

182

183

184

185

186

187

188

189

190

191

192

193

194

195

196

cKO) cells via flow cytometry. At 10 weeks post-transplant, recipient mice were euthanized and splenocytes were obtained for flow cytometry as described above.

through a 70uM filter, washed with MACS buffer, and mixed with 10\% Percoll in RPMI/PBS.

The samples in Percoll were spun at 2200rpm for 22min at 22C, with no brake or acceleration,

leading to isolation of lymphocytes in the pellet. These lymphocytes were then treated with RBC

Lysis Buffer to remove RBCs, and processed for flow cytometry as described above.

Isolation of lymphocytes from small intestine. To isolate lymphocytes from the small intestine, mice were euthanized, and following liver perfusion as described above, the entire small intestine was removed and placed in ice-cold media. The guts were cut open lengthwise to expose the lumen, and washed with ice-cold media. The tissue was then placed in a 50mL tube with $20 \mathrm{~mL}$ of strip buffer (containing 1x PBS, FBS, EDTA $0.5 \mathrm{M}$, and DTT 1M) and shaken at 37C for $30 \mathrm{~min}$. This removes the epithelium from the guts, so following incubation, the tissues were vortexed, supernatent was discarded, and the tissue was moved to a new tube. The gut was minced into small pieces, and $10 \mathrm{~mL}$ of digestion buffer was added to each tube (containing collagenase, DNAse, and RPMI). The tissues were incubated for $30 \mathrm{~min}$, shaking at 37C, and filtered into a clean tube using a 70uM filter. Remaining tissue pieces were ground onto the filter to obtain any remaining lymphocytes while leaving fat and structural cells behind. Finally, these cells were spun down in Percoll as described for livers, to isolate lymphocytes. RBC Lysis Buffer was not used on these cells. 
197 Cell culture for Treg markers. To test cells for the Treg markers (FOXP3, CD25, CTLA-4, IL-2,

198 IL-10), splenocytes were obtained from WT or TCF cKO naive mice. These cells were split into

199 three groups. Group 1 was simply stained for flow cytometry. Group 2 was cultured in LAK

200 media for 6 hours with Brefeldin A (GolgiPlug), in wells previously "coated” with PBS as a

201 control. Finally, Group 3 was cultured in LAK media for 6 hours with Brefeldin A in wells

202 coated with anti-CD3 in PBS (1ug/mL), and anti-CD28 $(2 \mathrm{ug} / \mathrm{mL})$ was added to the LAK media

203 for culture. After 6 hours of culture, all cells were collected from the stimulation plate and

204 stained for flow cytometry. All cells were stained for extracellular markers, then fixed overnight

205 with the Invitrogen Intracellular Fixation and Permeabilization buffer kit (cat. no. 88-8824-00).

206 The next day, the cells were permeabilized and stained with intracellular markers (FOXP3 for

207 all, IL-2 and IL-10 for cultured cells only). All samples were then run on a BD LSRFortessa flow

208 cytometer as described above.

209 Statistics. All statistics were performed using one-way ANOVA, two-way ANOVA, or Student's

210 t-test, depending on the dataset. ANOVA analyses included Tukey's multiple comparisons test.

211 P-values are presented as $<0.05$ being significant. Data graphing and statistical testing was

212 performed with GraphPad Prism v7 or v9 (GraphPad Software, San Diego, CA). Data are

213 presented as means with standard deviation. All experiments were done with at least 3 mice per

214 group, according to power analyses, and repeated multiple times.

\section{Results}

216 Loss of TCF-1 in all T cells leads to increased production of noncanonical Tregs. Delacher et

217 al. $[2020]^{24}$ recently claimed that loss of TCF-1 induces aberrant FOXP3 expression due to

218 release of suppressive control. However, this work was done with TCF-1 global KO mice or 
219 CRISPR-mediated deletion in T cells, giving potential off-target effects or developmental

220 changes in these cells. This work also did not address the role of TCF-1 in mature T cells.

221 Therefore, we sought to determine whether loss of TCF-1 specifically in mature T cells would

222 alter Treg populations. We obtained TCF-1 cKO mice which are deficient in TCF-1 in all T cells

223 following the DP stage of development. We phenotyped these TCF-1-deficient mice, and found

224 that loss of TCF-1 resulted in no change to canonical, FOXP3+ CD25+ Tregs [Fig 1A-B].

225 However, TCF cKO mice did have a significant increase in the frequency of noncanonical,

226 FOXP3 + CD25- Tregs [Fig 1A-B]. These cells were not increased in CD4cre control mice [Fig

227 1A], so the expansion of these cells was due to loss of TCF-1, not due to the CD4cre system for

228 deletion. Therefore, TCF-1 normally suppresses noncanonical Tregs, and loss of TCF-1 relieves

229 this suppression.

230

231

Downstream factors Eomes and T-bet do not impact noncanonical Treg frequency. TCF-1

232

controls the T cell downstream transcription factor Eomesodermin (Eomes), and may affect the

factor T-box transcription factor 21 (T-bet) through control of the $\mathrm{T}_{\mathrm{FH}} / \mathrm{Th} 1$ axis $^{32,40}$. To examine

whether changes in Eomes and T-bet during TCF-1 deficiency could play a role in the expansion 
242 Expansion of noncanonical Tregs due to TCF-1 deficiency is cell-intrinsic. Changes in

243 phenotype may be cell-intrinsic (due to loss of the factor in each individual cell) or cell-extrinsic

244 (from changes in the microenvironment in the mouse due to loss of the factor). We created bone

245 marrow chimeras to test whether the change to noncanonical Tregs was cell-intrinsic or not.

246 Briefly, bone marrow from WT and TCF cKO mice was mixed at a 1:4 (WT:TCF cKO) ratio.

247 The ratio was chosen based on our previous studies and observations that TCF cKO T cells do

248 not proliferate well. The bone marrow mixture was transplanted into irradiated Thy1.1 mice

249 (donor and host both on $\mathrm{H} 2 \mathrm{~Kb}$ background), and the mice were checked at 9 weeks by flow

250 cytometry on blood to ensure reconstitution. At 10 weeks, splenocytes were taken from these

251 mice and phenotyped by flow cytometry [Fig 2]. We found that TCF cKO donor T cells in the

252 chimeras still showed increased frequency of noncanonical Tregs, despite development in a

253 normal WT thymus [Fig 2]. Interestingly, the frequency of canonical Tregs was also increased in

254 TCF cKO donor cells in the chimeras [Fig 2]. These results show that TCF-1 cell-intrinsically

255 controls expansion of noncanonical Tregs.

256

257

TCF-1-deficient T cells induce increased production of WT noncanonical Tregs in a prion-

258 like manner. We performed the chimera experiment described above to determine whether the

259 effect of TCF-1 deficiency on noncanonical Tregs was cell-intrinsic. Given that the increase in

260 CD25- Tregs was cell-intrinsic, we next sought to determine whether the number of cells was

261 impacted along with the frequency of these cells. Loss of TCF-1 in this model results in a lower

262 frequency and number of CD4 T cells; thus, we wanted to know if the number of noncanonical 
263 Tregs was actually higher than in WT mice, given that the frequency is based out of CD4 T cells.

264 When we looked at the number of CD25+ FOXP3 + Tregs per 100,000 CD3 T cells in naïve WT

265 or TCF cKO mice, we found that the number of canonical Tregs was significantly decreased in

266 the TCF cKO mouse [Fig 3A]. This was expected given the drop in CD4 T cell numbers with no

267 difference in canonical Treg frequency [Fig 1B]. Interestingly, the number of noncanonical

268 (CD25- FOXP3+) Tregs was significantly higher in TCF cKO mice compared to WT mice [Fig

$2693 \mathrm{~A}]$, despite the reduction in CD4 T cells. Therefore, even among total $\mathrm{T}$ cells, noncanonical

270 Tregs make up a greater component of the T cells in TCF cKO mice than in WT mice. When we

271 looked at the cell numbers per 100,000 CD4+ T cells for naive mice, we saw an increase in

272 CD25- Treg numbers from TCF cKO mice, as expected based on the increased frequency out of

273 CD4 T cells [Fig 3B].

274

275

Next, we looked at the cell numbers for these Tregs in the chimeric mice. We calculated

276 the number of Treg cells per 100,000 CD3+ T cells of each donor type (because WT and TCF

277 cKO donor T cells were mixed in the mouse). In the chimera, we found no difference in the

278 numbers of canonical or noncanonical Tregs in these two donor types out of CD3 T cells [Fig

$2793 \mathrm{C}$ ]. However, the number of WT CD4 T cells in chimeric mice was nearly four times higher

280 than the number of TCF cKO CD4 T cells. This could result in an increased number of Tregs

281 seen among CD3 T cells even if the frequency of Tregs was the same for WT as for TCF cKO

282 mice, because the number of CD4 T cells was greater per 100,000 CD3 T cells. Therefore, we

283 looked at the number of Tregs per 100,000 CD4+ T cells, and we saw a reduced number of both

284 CD25+ and CD25- Tregs from WT donors compared to TCF cKO donors [Fig 3D]. However, 
285 the number of noncanonical Tregs derived from WT T cells in the chimera was increased

286 compared to the numbers found in the WT naive mice [Fig 3E,F], as was the frequency of these

287 cells [Fig 1A, 2B]. The number of WT-derived CD25- Tregs out of CD4 T cells was increased in

288 the chimera to the average numbers found in the TCF cKO naive mice [Fig 3F]. This is highly

289 important as it suggests that, when in a mixed environment with WT cells, the TCF cKO T cells

290 induce more WT T cells to adopt the noncanonical Treg phenotype, resulting in a higher

291 frequency and number of these Tregs than would occur in naive WT mice. Therefore, loss of

292 TCF-1 produces a prion-like ability in T cells to promote nearby cells to develop a Treg

293 phenotype.

294

295

Noncanonical Tregs are found at increased frequency in multiple tissues from TCF-deficient

296

mice. The noncanonical Treg population we observed in this model was identified in the spleen.

297 To determine whether these cells existed only in the spleen, or could be found in other organs,

298 we phenotyped lymphocytes from the spleen, thymus, liver, and small intestine of WT or TCF

299 cKO mice [Fig 4]. We found that noncanonical Tregs were present even in WT mice in all of the

300 tested organs [Fig 4A]. Significantly higher frequencies of noncanonical Tregs were observed in

301 the thymus, spleen, and liver of TCF cKO mice compared to WT mice [Fig 4B]. It was very

302 difficult to obtain total lymphocytes from the guts of TCF cKO mice [Fig 4A]. Thus, we were

303 unable to clearly determine whether noncanonical Tregs are present in higher frequency in the

304 guts. Therefore, noncanonical Tregs are present in multiple tissues in WT and TCF cKO mice,

305 with higher frequencies appearing when TCF-1 is lost. Additionally, the thymus of TCF cKO

306 mice showed an increase in canonical Tregs [Fig 4C]. Overall, these data show that CD25- Tregs 
307 are found in multiple tissues, with expansion due to loss of TCF-1 occurring in these peripheral 308 tissues as well.

309

310 Expansion of noncanonical Tregs is not due to aberrant FOXP3 expression. Delacher et al.

$311[2020]^{24}$ recently claimed that global deficiency of TCF-1 led to aberrant expression of FOXP3

312 in both CD4 and CD8 T cells, resulting in conventional T cells which appeared to be Tregs but

313 were not. To determine whether aberrant FOXP3 expression was the reason for expansion of

314 noncanonical Tregs, we first examined FOXP3 expression on CD8 T cells in WT and TCF cKO

315 mice [Fig 5]. We found that there was no significant increase in FOXP3 + CD25- cells among

316 CD8 T cells from TCF cKO naive mice [Fig 5A-B]. Therefore, aberrant expression of FOXP3 in

317 CD8 T cells is not occurring in this model of TCF-1 deletion.

Next, we examined whether CD25- Tregs from WT and TCF cKO expressed other

320 functional Treg markers or IL-2 [Fig 6]. Briefly, splenocytes from naive mice were obtained, and

321 split into three groups: one group was simply stained for flow cytometry, one was cultured for 6

322 hours with Brefeldin A in wells coated with PBS, then stained, and one was cultured for 6 hours

323 with Brefeldin A in wells coated with anti-CD3 and soluble anti-CD28 (in the media), then

324 stained. The uncultured samples were stained for CTLA-4, while the cultured cells were stained

325 for CTLA-4, IL-2, and IL-10. For CTLA-4 expression, no significant difference between CD25-

326 Tregs from WT or TCF cKO mice detected. However, Tregs from TCF cKO mice trended

327 towards increasing frequency of CTLA-4 expression for all conditions [Fig 6A]. No significant

328 difference was observed for IL-2 or IL-10 expression between WT and TCF cKO CD25- Tregs, 
329 but in both cases, the TCF cKO cells cultured with antibody stimulation trended toward

330 increased expression of these cytokines compared to WT cells [Fig 6B-C]. The level of IL-2

331 production was similar for TCF cKO CD25- Tregs compared to WT CD25- and CD25+ Tregs,

332 which are known to be suppressive [Fig 6B]. We did note the same trends for all three markers in

333 CD25+ Tregs, but the frequency of cells expressing these markers tended to be higher among

334 CD25+ than CD25- Tregs [Supp Fig 1]. This suggests that loss of TCF-1 may also enhance

335 expression of these markers in canonical Tregs. Therefore, the CD25- Tregs observed in WT and

336 expanded in TCF cKO mice are not simply the product of aberrant FOXP3 expression. These

337 cells express CTLA-4 and produce IL-2 and IL-10 at similar frequencies as in WT mice,

338 suggesting that they are truly noncanonical Tregs with suppressive function and not just

339 activated conventional T cells.

\section{Discussion}

341 TCF-1 is a major T cell transcription factor which controls $\mathrm{T}$ cell development and

342 lineage, and also has an important role in T cell responses to infection ${ }^{23,24,25,26,27,28,29,30,31,32,33,40 .}$

343 However, the role of TCF-1 in controlling mature T cells following normal development has not

344 been well studied. Our studies sought to determine whether loss of TCF-1 in mature T cells

345 (following development) would impact Treg cell fates in vivo in the mouse, given that TCF-1 is

346 known to repress FOXP3 expression ${ }^{24}$. Loss of TCF-1 in this context - using a Tcf $7^{\text {flox/lox }} \mathrm{x}$

347 CD4cre mouse - resulted in production of a smaller number and frequency of CD4 T cells, as

348 has been reported previously ${ }^{37}$. However, of the CD4 T cells present, there was a higher fraction

349 of noncanonical Tregs found in the TCF cKO mice compared to WT, while no change in

350 frequency was found for canonical Tregs. This suggests that the expression of FOXP3 in 
351 noncanonical cells from the TCF cKO mice may not simply be aberrant elevated expression of

352 FOXP3, because if that were the case, one would expect more canonical Tregs as well. In

353 addition, the expression of IL-10 and CTLA-4 at WT levels among CD25- Tregs suggests that

354 these expanded cells from TCF cKO mice are actually potentially suppressive Treg cells, not the

355 product of abnormal FOXP3 expression. This is in direct opposition to what Delacher et al.

$356[2020]^{24}$ reported from use of global TCF-1 KO mice and CRISPR-mediated deletion of TCF-1,

357 suggesting that TCF-1 may have a different role in mature cells than in developing cells.

Of note, our studies showed that the rise in noncanonical Tregs was cell-intrinsic, and not a result of microenvironment changes in the mouse due to loss of TCF-1, as may be more likely in a TCF-1 global $\mathrm{KO}^{37}$. In addition, TCF-1 controls Eomes and possibly T-bet expression ${ }^{32,40}$,

361 yet Eomes cKO and T-bet cKO mice showed no changes to noncanonical Tregs. This suggests

362 that TCF-1 and loss thereof is directly responsible for the expansion of CD25- FOXP3+ Tregs.

363 These noncanonical Tregs were found in small intestine, spleen, thymus, and liver, suggesting

364 that they are capable of migrating to potential sites of immune responses where suppression may

365 be needed. If these cells were simply aberrantly exiting the thymus without expression CD25, we

366 would expect the frequency of these cells to be higher in the thymus than other organs, but this

367 was not the case. In fact, the frequency of these cells was higher in liver, guts, and spleen than in

368 thymus, especially for the TCF cKO mice. Therefore, these noncanonical Tregs appear to be

369 stable peripheral Tregs which have lost CD25 expression at some point after exiting the 370 thymus $^{13}$. 
372 chimera, which involved mixing BM cells from WT and TCF cKO mice and allowing them to

373 develop to maturity in a WT thymus. We then examined frequency of CD25+ and CD25- Tregs

374 in the spleen. This allowed us to determine that the expansion of CD25- Tregs is cell-intrinsic,

375 because the rise in frequency of these cells for TCF cKO mice compared to WT mice was

376 preserved for this model. Surprisingly, when we looked at the number of CD25+/- Tregs per

$377100,000 \mathrm{CD} 3 \mathrm{~T}$ cells of each donor type in this model, we saw no difference for either type of

378 Treg. We thought that this could be because the number of CD4 T cells from WT donors was

379 almost four times as high as the number of CD4 T cells from the TCF cKO donor, within each

380 chimeric mouse. Even if the frequency of CD25- Tregs was less in WT mice, this could result in

381 similar cell numbers when normalized to CD3 T cells. Therefore, we looked at cell numbers per

$382100,000 \mathrm{CD} 4 \mathrm{~T}$ cells. We first saw that the number of TCF cKO CD25+ Tregs in the chimera

383 increased compared to in TCF cKO naive mice, so that the number of canonical Tregs was

384 significantly higher than those from WT donors. This suggested that being in a WT environment

385 induced higher production of these cells from TCF cKO cells. Unsurprisingly, we also saw that

386 the number of CD25- Tregs was less for WT donors than for TCF cKO donors, similar to the

387 frequency differences. However, we found that this was because the presence of TCF-1-deficient

388 T cells had induced production of higher numbers of noncanonical Tregs from the WT donor

389 BM than would occur in a naive WT mouse. We also saw that the frequency of these cells from

390 WT donors was increased in the chimera compared to among naive WT T cells. This is critical as

391 it suggests that loss of TCF-1 in T cells can alter the microenvironment (regardless of the fact 
392 that the cells in the microenvironment are WT), leading to alterations in the fate decisions and 393 phenotype of nearby cells.

This trait is similar to the ability of pathogenic prion proteins to induce misfolding of

395 nearby normal prion proteins into the pathogenic state ${ }^{41}$. In a similar manner, despite the WT

396 environment, TCF-deficient T cells are able to induce more production of CD25- Tregs from WT

397 donors cells. This finding - and this trait generally - could have a major impact on attempts to

398 raise yield of suppressive cells for therapeutic purposes. It also shows a novel way by which

399 deficiency within a cell could impact nearby cells, most likely by release of factors into the

400

microenvironment which can alter fate decisions. To our knowledge, the ability of gene- or gene-

401 product-deficient cells to alter WT cells in a WT environment in this fashion has not yet been

402 described, so this trait is a major novel finding.

403

Interestingly, the number of canonical Tregs was lower in TCF cKO mice when

404 normalized to the amount of CD3 T cells, which makes sense given the drop in CD4 T cells

405 numbers in these mice. However, despite this drop in CD4 cells, the number of noncanonical

406 Tregs was still higher in TCF cKO mice compared to WT mice. This suggests that loss of TCF-1

407 increases the yield of cells adopting a suppressive fate, while potentially disrupting the

408 expression of CD25, given that fewer CD25+ and more CD25- cells were observed. CD25 is part

409 of the IL-2 receptor ${ }^{42}$, so the reduced expression of CD25 suggests that the Tregs expanded by

410 loss of TCF-1 may not be as receptive to IL-2 stimulation, possibly providing a clue as to the

411 apparent reduction in proliferation or survival of $\mathrm{T}$ cells from TCF cKO donors in the chimera

412 model. 
Overall, these results show that TCF-1 normally suppresses FOXP3 in CD4 T cells, and

414 that loss of TCF-1-driven suppression allows expansion of CD25-FOXP3+ Tregs. These cells

415 also express other markers found on functional Tregs, including IL-10, and CTLA-4, indicating

416 they are true Tregs with the potential to be suppressive. The presence of TCF-deficient $\mathrm{T}$ cells in

417 a WT environment induced increased production of these unique Treg from WT donor cells,

418 showing a prion-like ability of TCF cKO T cells to control cell fate in neighboring cells in the

419 microenvironment. These CD25- Tregs are found in multiple tissues, suggesting they may be

420 stable Tregs rather than a product of transient aberrant FOXP3 expression. CD8 cells expressing

421 FOXP3 but not CD25 were not increased by loss of TCF-1, further showing that these cells are

422 not due to aberrant FOXP3 expression. In all, these data suggest that TCF-1 normally suppresses

423 FOXP3 to control expansion of this CD25- Treg subset, and modulation of TCF-1 may provide

424 an opportunity to increase production of these cells.

425 Future work should address the functional capacity of these Treg cells, although the

426 suppressive ability of CD25- Tregs from normal WT mice has been proven in several

427 experimental models ${ }^{11,12,14}$. The mechanism or factors by which TCF-deficient cells evoke fate

428 changes in WT cells in a mixed cell model should also be examined, as this prion-like ability

429 could have significant impacts on cell culture strategies for therapeutic uses.

\section{Author Contributions}

$431 \mathrm{RH}$ and MK designed the experiments, analyzed the data, and wrote the manuscript. RH

432 performed all experiments. MM assisted with experiments. MK assisted with scientific and

433 technical design and discussion. 
435 Acknowledgments

436 We would like to thank Dr. Jyoti Misra Sen from the NIH for providing us with TCF cKO mice

437 to start our colony. We would also like to thank members of the Karimi lab for their helpful

438 discussions, and members of the Yang lab for proofreading this manuscript.

439 Funding: This research was funded in part by a grant from the National Blood Foundation

440 Scholar Award to (MK) and the National Institutes of Health (NIH LRP \#L6 MD0010106 and

441 AI130182 to MK.

442

443 Conflict of interest statement: The authors have declared that no conflict of interest exists.

444

445

446

447

448

449

450

451

452

453 


\section{References}

455 1. Li, Y. \& Mariuzza, R. Structural and Biophysical Insights into the Role of CD4 and CD8

456 in T Cell Activation. Frontiers in Immunology 4 (2013).

457

458

459

2. Xing, Y. \& Hogquist, K.A. T-cell tolerance: central and peripheral. Cold Spring Harb

460

461

462 Perspect Biol 4, a006957 (2012).

463

464

465

466

3. Kappler, J.W., Roehm, N. \& Marrack, P. T cell tolerance by clonal elimination in the thymus. Cell 49, 273-280 (1987).

467

468

5. ElTanbouly, M.A. \& Noelle, R.J. Rethinking peripheral T cell tolerance: checkpoints across a T cell's journey. Nature Reviews Immunology (2020).

470

471 6. Jørgensen, N., Persson, G. \& Hviid, T.V.F. The Tolerogenic Function of Regulatory T Cells in Pregnancy and Cancer. Frontiers in Immunology 10 (2019).

473

474 7. Brunkow, M.E., Jeffery, E.W., Hjerrild, K.A., Paeper, B., Clark, L.B., Yasayko, S.A., 475 Wilkinson, J.E., Galas, D., Ziegler, S.F. \& Ramsdell, F. Disruption of a new 
476

477

478

479 8. Godfrey, V.L., Wilkinson, J.E. \& Russell, L.B. X-linked lymphoreticular disease in the 480

481

482

483

484

485

486

487

488

489

490

491

492

493

494 12. Coleman, M.M., Finlay, C.M., Moran, B., Keane, J., Dunne, P.J. \& Mills, K.H. The 495

496

497 forkhead/winged-helix protein, scurfin, results in the fatal lymphoproliferative disorder of the scurfy mouse. Nat Genet 27, 68-73 (2001).
9. Ochs, H.D., Gambineri, E. \& Torgerson, T.R. IPEX, FOXP3 and regulatory T-cells: a model for autoimmunity. Immunol Res 38, 112-121 (2007).

10. Sakaguchi, S., Wing, K., Onishi, Y., Prieto-Martin, P. \& Yamaguchi, T. Regulatory T cells: how do they suppress immune responses? International Immunology 21, 1105-1111 (2009).

11. Angerami, M.T., Suarez, G.V., Vecchione, M.B., Laufer, N., Ameri, D., Ben, G., Perez, H., Sued, O., Salomón, H. \& Quiroga, M.F. Expansion of CD25-Negative Forkhead Box P3-Positive T Cells during HIV and Mycobacterium tuberculosis Infection. Frontiers in Immunology 8 (2017). immunoregulatory role of $\mathrm{CD} 4^{+} \mathrm{FoxP}^{+} \mathrm{CD} 25^{-}$regulatory $\mathrm{T}$ cells in lungs of mice infected with Bordetella pertussis. FEMS Immunol Med Microbiol 64, 413-424 (2012). 
498 13. Curotto de Lafaille, M.A., Lino, A.C., Kutchukhidze, N. \& Lafaille, J.J. CD25- T cells generate CD25+Foxp3+ regulatory T cells by peripheral expansion. J Immunol 173,

500 7259-7268 (2004).

501

502 14. Annacker, O., Pimenta-Araujo, R., Burlen-Defranoux, O., Barbosa, T.C., Cumano, A. \& Bandeira, A. CD25- CD4+ T Cells Regulate the Expansion of Peripheral CD4 T Cells Through the Production of IL-10. The Journal of Immunology 166, 3008 (2001).

505

506 15. Apostolou, I., Sarukhan, A., Klein, L. \& von Boehmer, H. Origin of regulatory T cells with known specificity for antigen. Nat Immunol 3, 756-763 (2002).

508

509

16. Furtado, G.C., Olivares-Villagómez, D., Curotto de Lafaille, M.A., Wensky, A.K., encephalomyelitis. Immunol Rev 182, 122-134 (2001).

513 17. Stephens, L.A. \& Mason, D. CD25 Is a Marker for CD4+ Thymocytes That Prevent Autoimmune Diabetes in Rats, But Peripheral T Cells with This Function Are Found in (2000). 
518 18. Dowling, M.R., Kan, A., Heinzel, S., Marchingo, J.M., Hodgkin, P.D. \& Hawkins, E.D.

519 Regulatory T Cells Suppress Effector T Cell Proliferation by Limiting Division Destiny.

$520 \quad$ Front Immunol 9, 2461 (2018).

521

522 19. Jain, N., Nguyen, H., Chambers, C. \& Kang, J. Dual function of CTLA-4 in regulatory T 523 cells and conventional T cells to prevent multiorgan autoimmunity. Proceedings of the $524 \quad$ National Academy of Sciences 107, 1524 (2010).

525

526 20. Verhagen, J., Gabryšová, L., Shepard, E.R. \& Wraith, D.C. CTLA-4 Modulates the 527 Differentiation of Inducible Foxp3+ Treg Cells but IL-10 Mediates Their Function in 528 Experimental Autoimmune Encephalomyelitis. PLOS ONE 9, e108023 (2014).

529

530 21. Zhao, H., Liao, X. \& Kang, Y. Tregs: Where We Are and What Comes Next? Frontiers $531 \quad$ in Immunology 8 (2017).

532

533 22. Wan, Y.Y. \& Flavell, R.A. 'Yin-Yang' functions of transforming growth factor-beta and 534 T regulatory cells in immune regulation. Immunological reviews 220, 199-213 (2007).

535

536 23. Yang, B.H., Wang, K., Wan, S., Liang, Y., Yuan, X., Dong, Y., Cho, S., Xu, W., Jepsen, 537 K., Feng, G.S., Lu, L.F., Xue, H.H. \& Fu, W. TCF1 and LEF1 Control Treg Competitive 538 Survival and Tfr Development to Prevent Autoimmune Diseases. Cell Rep 27, 3629$539 \quad 3645 . e 3626(2019)$. 
541 24. Delacher, M., Barra, M.M., Herzig, Y., Eichelbaum, K., Rafiee, M.-R., Richards, D.M.,

542 Träger, U., Hofer, A.-C., Kazakov, A., Braband, K.L., Gonzalez, M., Wöhrl, L.,

543 Schambeck, K., Imbusch, C.D., Abramson, J., Krijgsveld, J. \& Feuerer, M. Quantitative

544 Proteomics Identifies TCF1 as a Negative Regulator of Foxp3 Expression in

545 Conventional T Cells. iScience 23, 101127-101127 (2020).

546

547 25. Chen, Z., Ji, Z., Ngiow, S.F., Manne, S., Cai, Z., Huang, A.C., Johnson, J., Staupe, R.P., 548 Bengsch, B., Xu, C., Yu, S., Kurachi, M., Herati, R.S., Vella, L.A., Baxter, A.E., Wu, 549 J.E., Khan, O., Beltra, J.C., Giles, J.R., Stelekati, E., McLane, L.M., Lau, C.W., Yang, 550 X., Berger, S.L., Vahedi, G., Ji, H. \& Wherry, E.J. TCF-1-Centered Transcriptional $551 \quad$ Network Drives an Effector versus Exhausted CD8 T Cell-Fate Decision. Immunity 51, $552 \quad$ 840-855.e845 (2019).

553

554 26. Johnson, J.L., Georgakilas, G., Petrovic, J., Kurachi, M., Cai, S., Harly, C., Pear, W.S., 555 Bhandoola, A., Wherry, E.J. \& Vahedi, G. Lineage-Determining Transcription Factor 556 TCF-1 Initiates the Epigenetic Identity of T Cells. Immunity 48, 243-257.e210 (2018).

557

558 27. Kim, C., Jin, J., Weyand, C.M. \& Goronzy, J.J. The Transcription Factor TCF1 in T Cell 559 Differentiation and Aging. Int J Mol Sci 21 (2020). 
561 28. Rutishauser, R.L., Deguit, C.D.T., Hiatt, J., Blaeschke, F., Roth, T.L., Wang, L.,

562

563

564

565

566

567

568

569

570

571

572

573 30. Welten, S.P.M., Yermanos, A., Baumann, N.S., Wagen, F., Oetiker, N., Sandu, I.,

574

575

576

577

578 31. Yu, Q., Sharma, A. \& Sen, J.M. TCF1 and beta-catenin regulate T cell development and 579 580

Raymond, K., Starke, C.E., Mudd, J.C., Chen, W., Smullin, C., Matus-Nicodemos, R., Hoh, R., Krone, M., Hecht, F.M., Pilcher, C.D., Martin, J.N., Koup, R.A., Douek, D.C., Brenchley, J.M., Sékaly, R.-P., Pillai, S.K., Marson, A., Deeks, S.G., McCune, J.M. \& Hunt, P.W. TCF-1 regulates the stem-like memory potential of HIV-specific CD8+ T cells in elite controllers. bioRxiv, 2020.2001.2007.894535 (2020).

29. Wang, Y., Hu, J., Li, Y., Xiao, M., Wang, H., Tian, Q., Li, Z., Tang, J., Hu, L., Tan, Y., Zhou, X., He, R., Wu, Y., Ye, L., Yin, Z., Huang, Q. \& Xu, L. The Transcription Factor TCF1 Preserves the Effector Function of Exhausted CD8 T Cells During Chronic Viral Infection. Frontiers in Immunology 10 (2019). Pedrioli, A., Oduro, J.D., Reddy, S.T., Cicin-Sain, L., Held, W. \& Oxenius, A. Tcf1+ cells are required to maintain the inflationary T cell pool upon MCMV infection. Nature Communications 11, 2295 (2020). function. Immunol Res 47, 45-55 (2010). 
581 32. Zhou, X., Yu, S., Zhao, D.M., Harty, J.T., Badovinac, V.P. \& Xue, H.H. Differentiation 582 and persistence of memory CD8(+) T cells depend on T cell factor 1 . Immunity 33, 229$583240(2010)$

584

585 33. Verbeek, S., Izon, D., Hofhuis, F., Robanus-Maandag, E., te Riele, H., van de Wetering, M., Oosterwegel, M., Wilson, A., MacDonald, H.R. \& Clevers, H. An HMG-box-

587 containing T-cell factor required for thymocyte differentiation. Nature 374, 70-74 (1995).

\section{8}

589

34. Cho, S.W., Kim, S., Kim, Y., Kweon, J., Kim, H.S., Bae, S. \& Kim, J.-S. Analysis of offtarget effects of CRISPR/Cas-derived RNA-guided endonucleases and nickases. Genome

591 Res 24, 132-141 (2014).

592

593 35. Wang, Y., Wang, M., Zheng, T., Hou, Y., Zhang, P., Tang, T., Wei, J. \& Du, Q. Specificity profiling of CRISPR system reveals greatly enhanced off-target gene editing. Scientific Reports 10, 2269 (2020).

596

597 36. Zhang, X.-H., Tee, L.Y., Wang, X.-G., Huang, Q.-S. \& Yang, S.-H. Off-target Effects in 598 CRISPR/Cas9-mediated Genome Engineering. Molecular Therapy - Nucleic Acids 4, 599 e264 (2015)

600

601 37. Steinke, F.C., Yu, S., Zhou, X., He, B., Yang, W., Zhou, B., Kawamoto, H., Zhu, J., Tan, 602 K. \& Xue, H.-H. TCF-1 and LEF-1 act upstream of Th-POK to promote the CD4(+) T 
606 38. Ariga, H., Shimohakamada, Y., Nakada, M., Tokunaga, T., Kikuchi, T., Kariyone, A., 221 (2007).

610

611 39. Jenner, R.G., Townsend, M.J., Jackson, I., Sun, K., Bouwman, R.D., Young, R.A., alternative pathways of T-cell differentiation through a shared set of target genes. Proceedings of the National Academy of Sciences 106, 17876 (2009).

615

616 40. Shao, P., Li, F., Wang, J., Chen, X., Liu, C. \& Xue, H.-H. Cutting Edge: Tcf1 Instructs T 617 Follicular Helper Cell Differentiation by Repressing Blimp1 in Response to Acute Viral 618 Infection. The Journal of Immunology 203, 801 (2019).

620 41. Kupfer, L., Hinrichs, W. \& Groschup, M.H. Prion protein misfolding. Curr Mol Med 9 , 621 826-835 (2009). 
bioRxiv preprint doi: https://doi.org/10.1101/2021.03.11.435008; this version posted March 12, 2021. The copyright holder for this preprint (which was not certified by peer review) is the author/funder. All rights reserved. No reuse allowed without permission.

623 42. Létourneau, S., Krieg, C., Pantaleo, G. \& Boyman, O. IL-2- and CD25-dependent

624 immunoregulatory mechanisms in the homeostasis of T-cell subsets. J Allergy Clin

$625 \quad$ Immunol 123, 758-762 (2009).

626

627

628 
629 FIGURE LEGENDS

630 Fig 1. Loss of TCF-1 in T cells leads to increased production of noncanonical Tregs, with no

631 effect by Eomes and T-bet. Splenocytes were taken from naive mice and stained for CD4,

632 CD25, and FOXP3 to identify Treg populations. Canonical Tregs are CD4+ CD25+ FOXP3+,

633 and noncanonical Tregs are CD4+CD25- FOXP3+. (A) Flow cytometry plots of Treg

634 frequencies in WT, CD4cre heterozygous control, CD4cre homozygous control, and TCF cKO

635 naive mice, shown out of CD4 T cells. One representative plot is shown per group. (B)

636 Quantification of (A), with CD4cre groups not shown. (C) Flow cytometry plots of Treg

637 frequencies in WT, T-bet cKO, or Eomes cKO naive mice, shown out of CD4 T cells. One

638 representative plot is shown per group. (D) Quantification of (C), showing all groups. For all

639 graphs, $\mathrm{n}=3$ per group and one representative experiment is shown, mean and SD are also

640 plotted. P-values: $* * * *=p \leq 0.0001$, and N.S. $(\mathrm{p}>0.05)$ is not shown.

641

642 Fig 2. Expansion of noncanonical Tregs due to TCF-1 deficiency is cell-intrinsic. Bone

643 marrow cells from naive WT and TCF cKO mice were obtained and mixed at a 4:1 (TCF:WT)

644 ratio, and injected into lethally irradiated Thy 1.1 mice. At 10 weeks post-transplant, flow

645 cytometry was performed to look at Treg markers (CD25, FOXP3). (A) Flow cytometry plots of

646 Treg frequencies from WT donor-derived or TCF cKO donor-derived CD4 T cells within the

647 chimeric mouse. One representative plot is shown per group. (B) Quantification of (A). For all

648 graphs, $\mathrm{n}=5$ per group and one representative experiment is shown, mean and SD are also

649 plotted. P-values: $* *=\mathrm{p} \leq 0.01, * * *=\mathrm{p} \leq 0.001$, and N.S. $(\mathrm{p}>0.05)$ is not shown. 


\section{Fig 3. TCF-1-deficient $\mathbf{T}$ cells induce increased production of WT noncanonical Tregs in a}

652 prion-like manner. Splenocytes from WT or TCF cKO naive or chimeric mice were obtained

653 and stained for flow cytometry (data shown in Fig 1 and Fig 2, respecitvely). Here, cell numbers

654 are shown from these experiments. (A) Number of CD25+ or CD25- Tregs calculated per

655100,000 CD3 + T cells for naive WT or TCF cKO mice. (B) Number of CD25+ or CD25- Tregs

656 calculated per 100,000 CD4 T cells for naive WT or TCF cKO mice. (C) Number of CD25+ or

657 CD25- Tregs calculated per 100,000 CD3+ WT or TCF cKO donor-derived T cells within

658 chimeric mice. (D) Number of CD25+ or CD25- Tregs calculated per 100,000 CD4+ WT or TCF

659 cKO donor-derived T cells within chimeric mice. (E) Data from (A) and (C) combined, showing

660 number of CD25+ or CD25- Tregs calculated per 100,000 CD3+ T cells from WT or TCF cKO

661 donor-derived T cells within chimeric mice, or from WT or TCF cKO naive mice. (F) Data from

662 (B) and (D) combined, showing number of CD25+ or CD25- Tregs calculated per 100,000 CD4+

663 T cells from WT or TCF cKO donor-derived T cells within chimeric mice, or from WT or TCF

664 cKO naive mice. Data quantitated from flow cytometry plots. For all graphs, $\mathrm{n}=3$ to 5 per group

665 and one representative experiment is shown, mean and SD are also plotted. P-values: $*=p \leq 0.05$,

$666 * *=\mathrm{p} \leq 0.01, * * *=\mathrm{p} \leq 0.001, * * * *=\mathrm{p} \leq 0.0001$, and N.S. $(\mathrm{p}>0.05)$ is not shown.

667

668 Fig 4. Noncanonical Tregs are found at increased frequency in multiple tissues from TCF-

669 deficient mice. Lymphocytes were obtained from the spleen, thymus, small intestine ("gut"), and

670 liver as described in detail in the Methods section. These cells were taken from WT or TCF cKO

671 naive mice, and stained for flow cytometry. (A) Flow cytometry plots of Treg frequencies in

672 tissues of WT or TCF cKO naive mice, shown out of CD4 T cells. One representative plot is 
673 shown per group. (B,C) Quantification of (A), for canonical Tregs (B) and noncanonical Tregs

674 (C). For all graphs, $n=3$ per group and one representative experiment is shown, mean and SD are 675 also plotted. P-values: $*=\mathrm{p} \leq 0.05, * *=\mathrm{p} \leq 0.01, * * *=\mathrm{p} \leq 0.001$, and N.S. $(\mathrm{p}>0.05)$ is not shown.

676

677 Fig 5. Aberrant FOXP3 expression is not visible in CD8 T cells. Splenocytes were taken from 678 naive mice and either directly stained or cultured for 6 hours with PBS or anti-CD3/anti-CD28 679 stimulation prior to staining for CD4, CD8, CD25, and FOXP3. CD8 T cells were examined for 680 expression of FOXP3 and CD25 to identify aberrant expression. (A) Flow cytometry plots of 681 “Treg” frequencies in WT or TCF cKO naive mice, shown out of CD8 T cells. One 682 representative plot is shown per group. (B) Quantification of (A), showing noncanonical "Treg" 683 frequencies for each of the three conditions. For all graphs, $n=3$ per group and one representative 684 experiment is shown, mean and SD are also plotted. N.S. ( $p>0.05)$ is not shown, and no 685 significance was reported for this experiment.

686

687 Fig 6. Expansion of noncanonical Tregs is not due to aberrant FOXP3 expression.

688 Splenocytes were taken from naive mice and either directly stained or cultured for 6 hours with 689 PBS or anti-CD3/anti-CD28 stimulation prior to staining for CTLA-4, IL-2, and IL-10. CD25690 Tregs were examined for expression of these Treg markers. Flow cytometry plots not shown. (A) 691 Quantification of CTLA-4 expression among CD25- Tregs for uncultured, control (PBS) 692 cultured, and antibody-stimulated cultured T cells. (B) Quantification of IL-2 expression among 693 CD25- Tregs for control (PBS) cultured and antibody-stimulated cultured T cells. (C)

694 Quantification of IL-10 expression among CD25- Tregs for control (PBS) cultured and antibody- 
695 stimulated cultured T cells. Data quantitated from flow cytometry plots. For all graphs, $\mathrm{n}=3$ per

696 group and one representative experiment is shown, mean and SD are also plotted. N.S. (p>0.05)

697 is not shown, and no significance was reported for this experiment. 


\section{Summary Figure}

\section{Wnt $\beta$-catenin pathway}

(which was not certified by peer review) is the author/funder. All rights reserved. No reuse allowed without permission.
(1) preprint

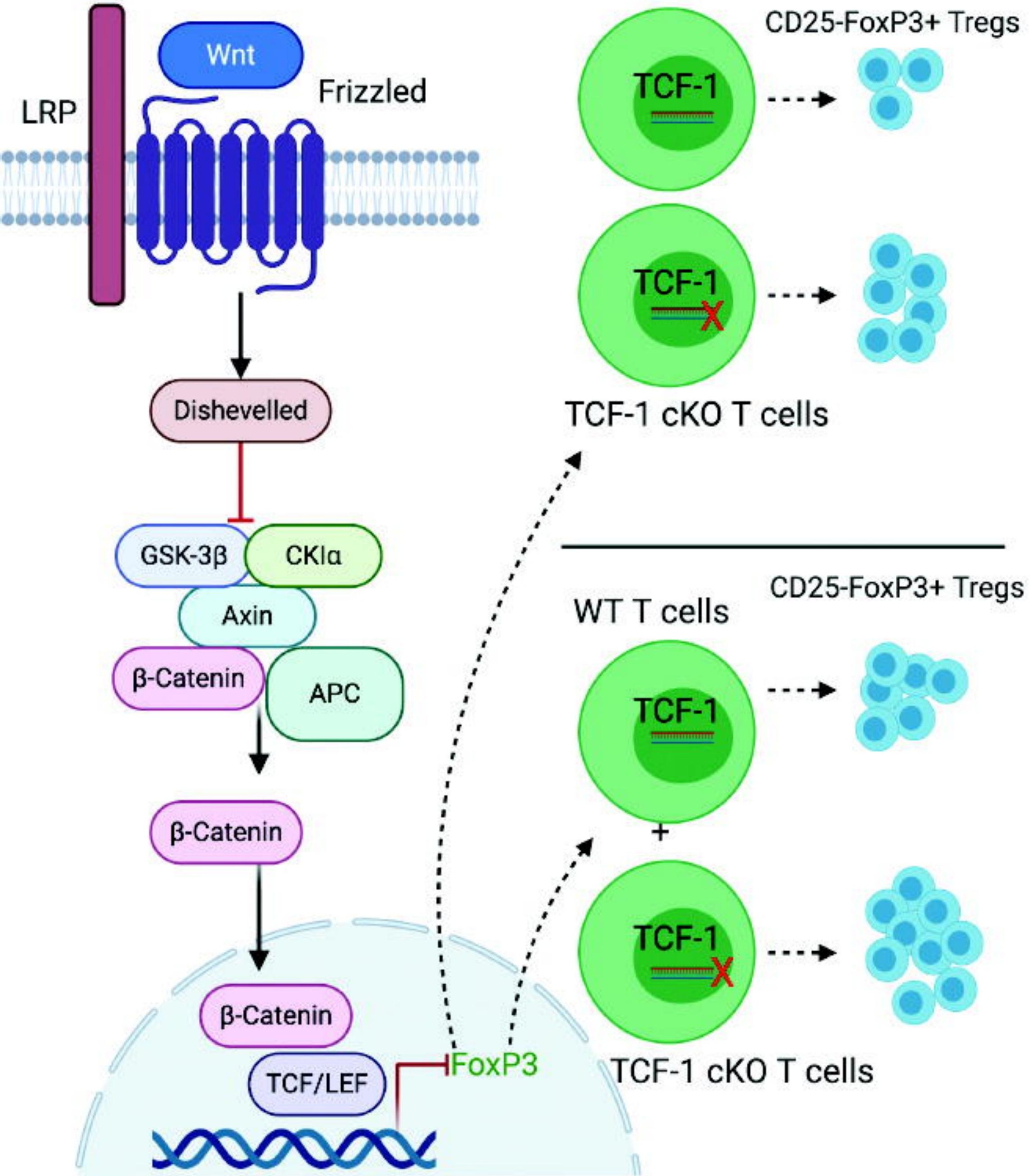


Fig 1

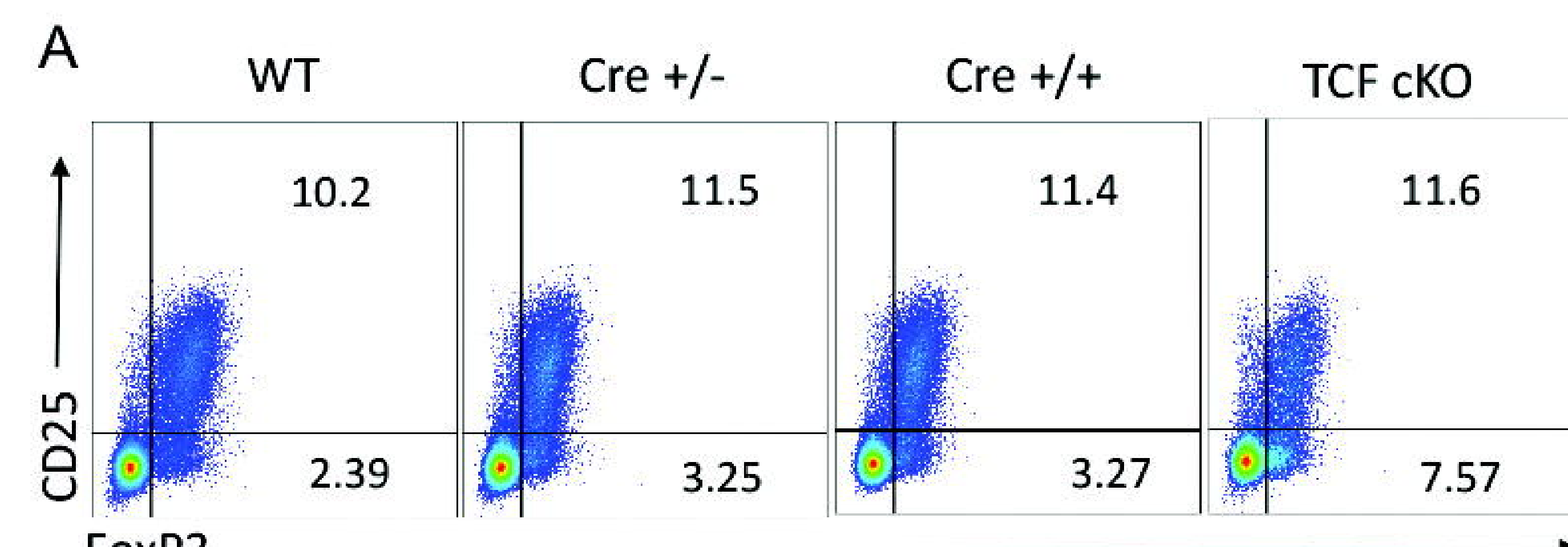

FoxP3

B

ڤ̆

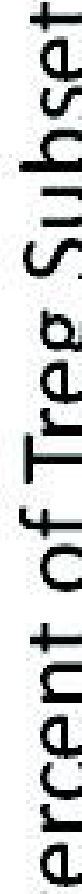

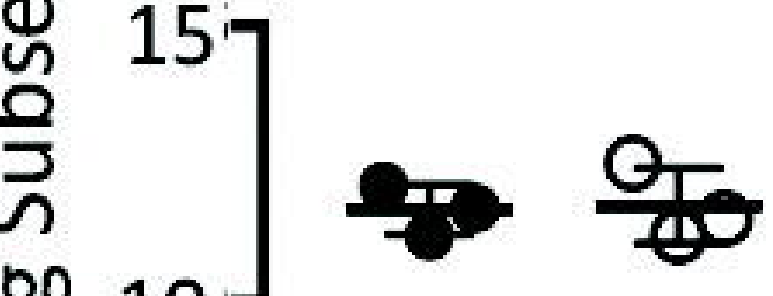

บํํㅇ
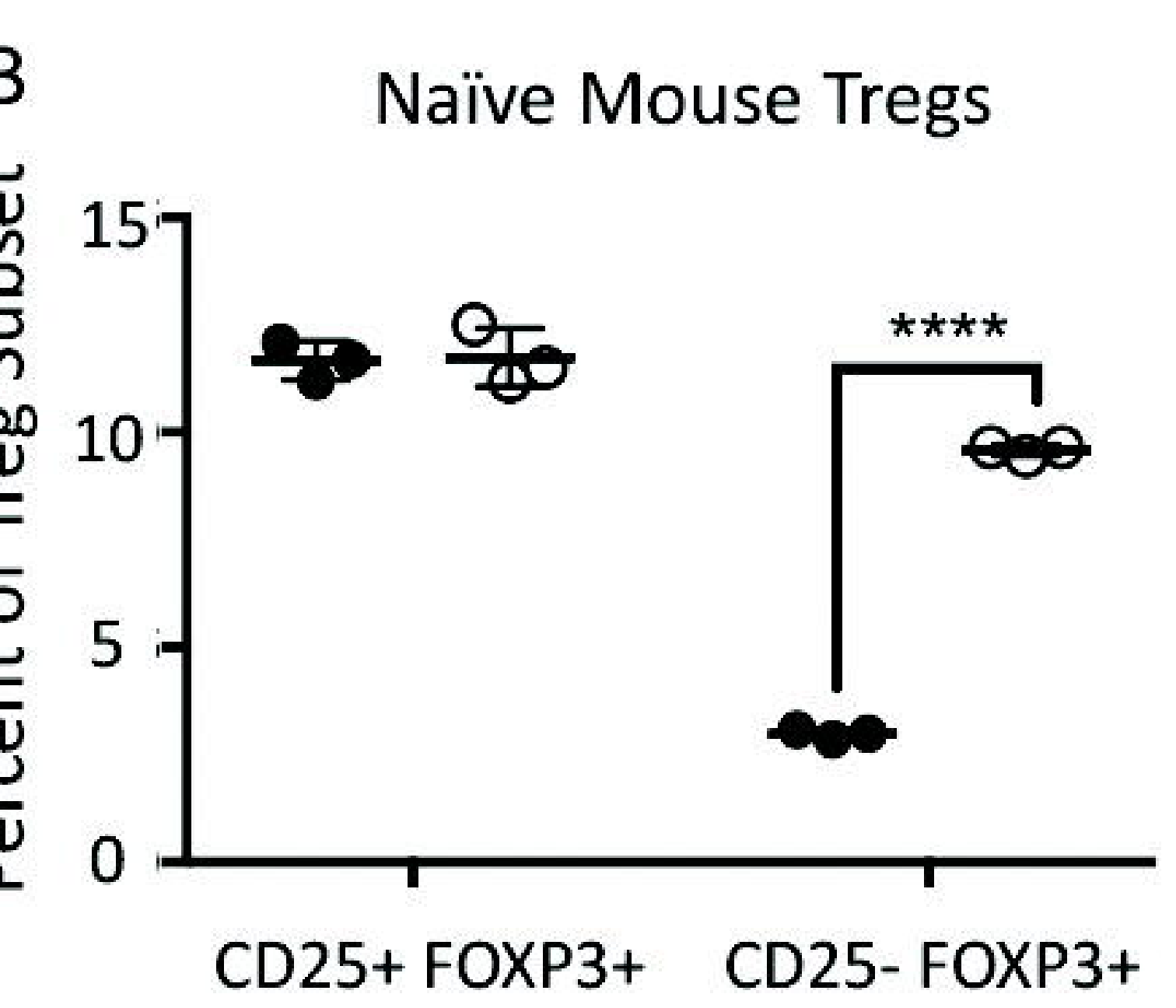

D

- WT

O TCF-1 KO

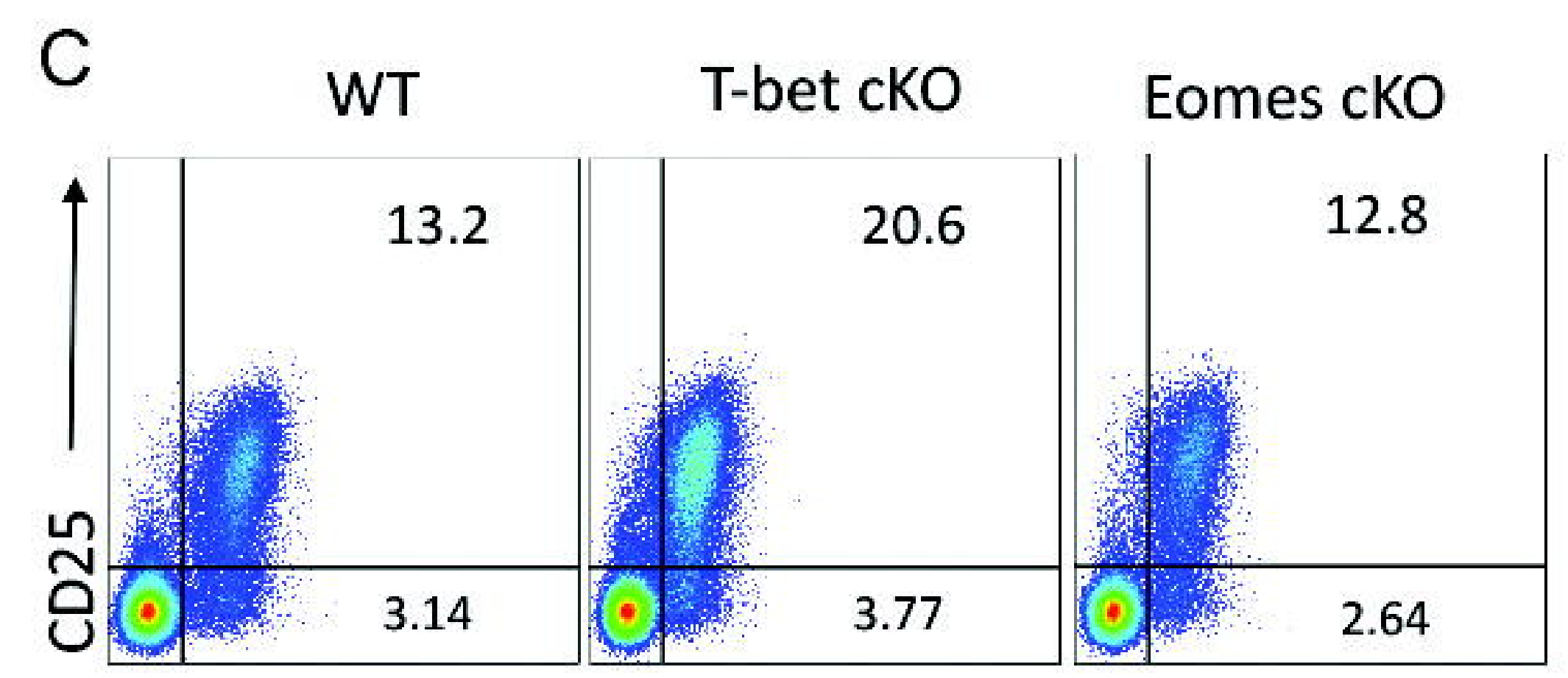

FoxP3

Naïve Mouse Tregs

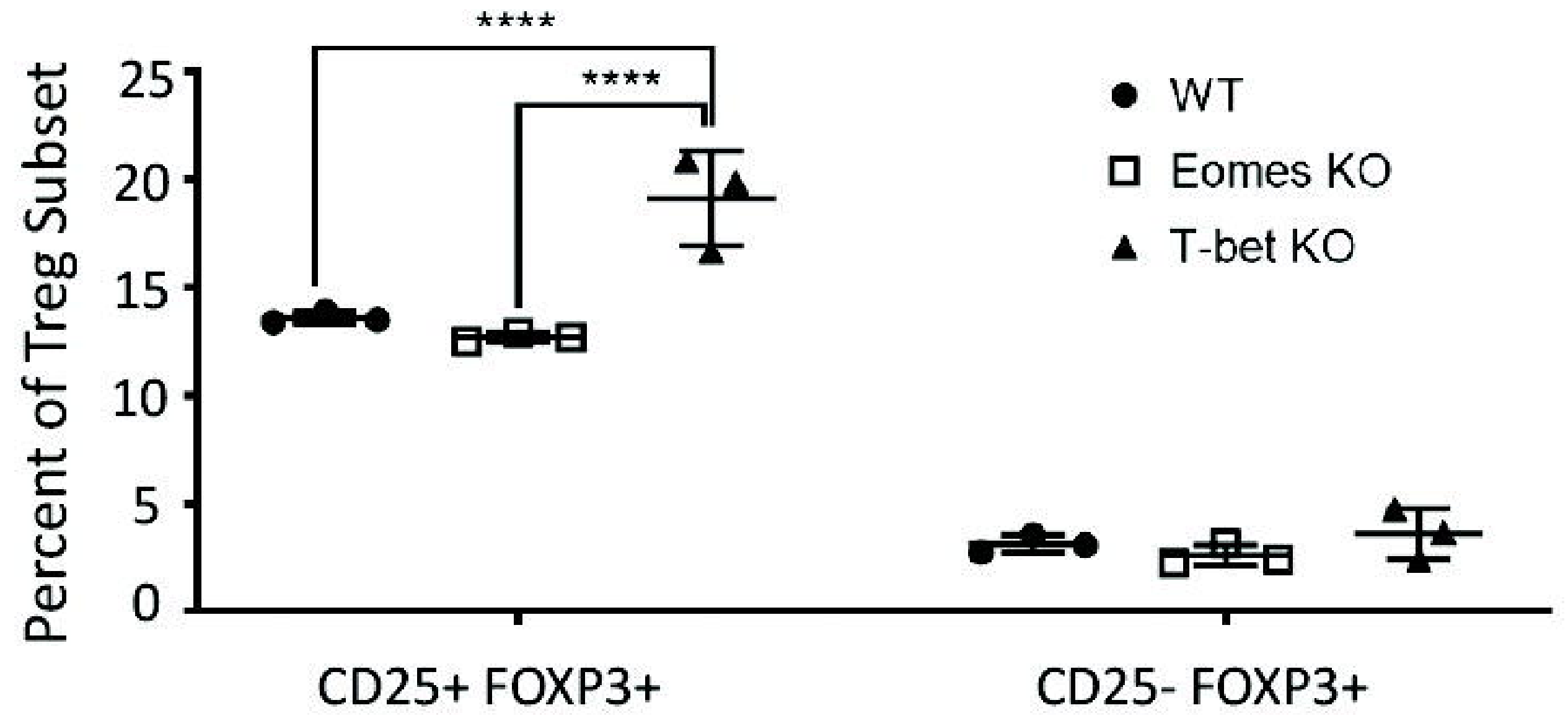


Fig 2

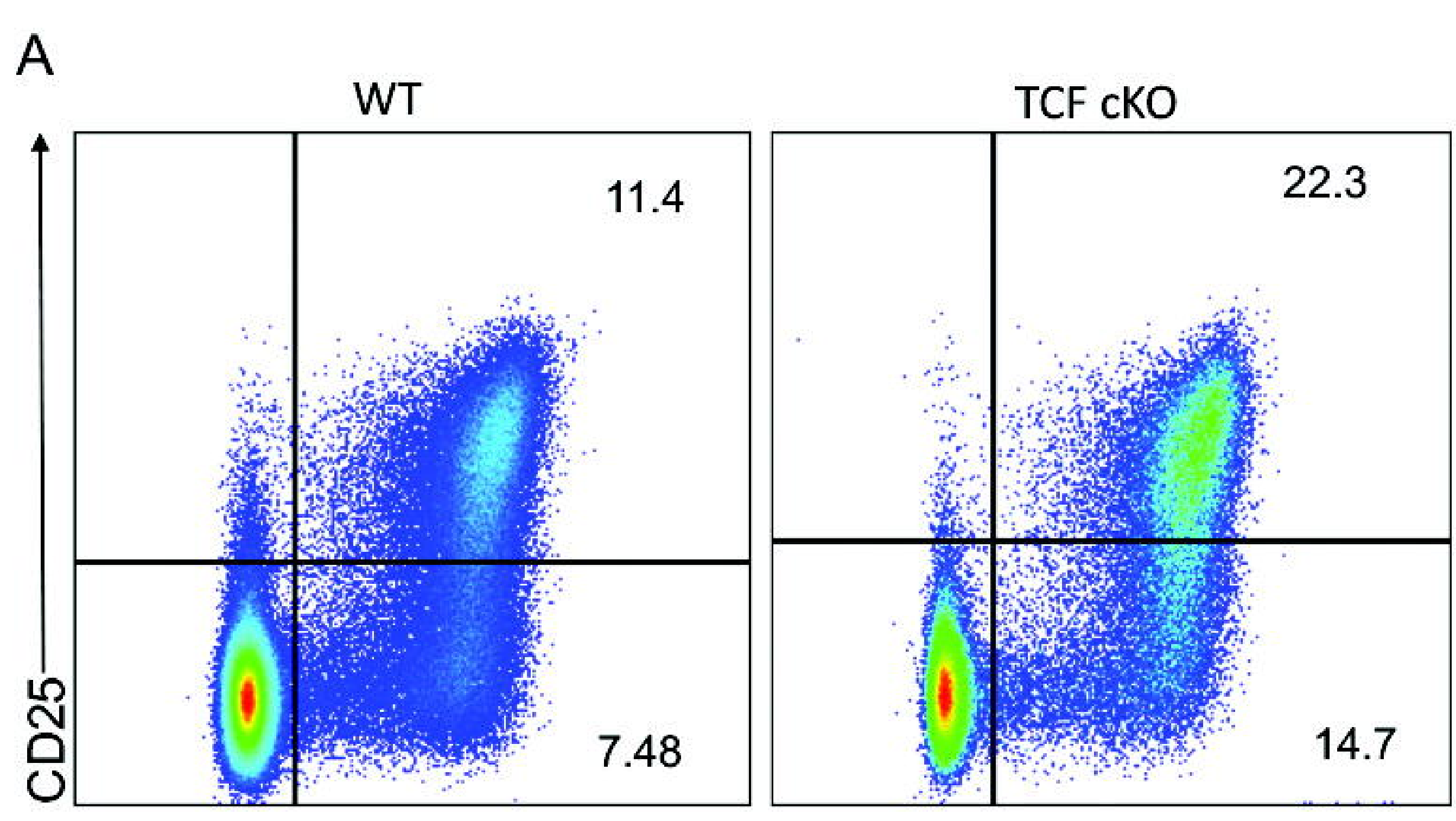

FoxP3
B

Chimera Tregs

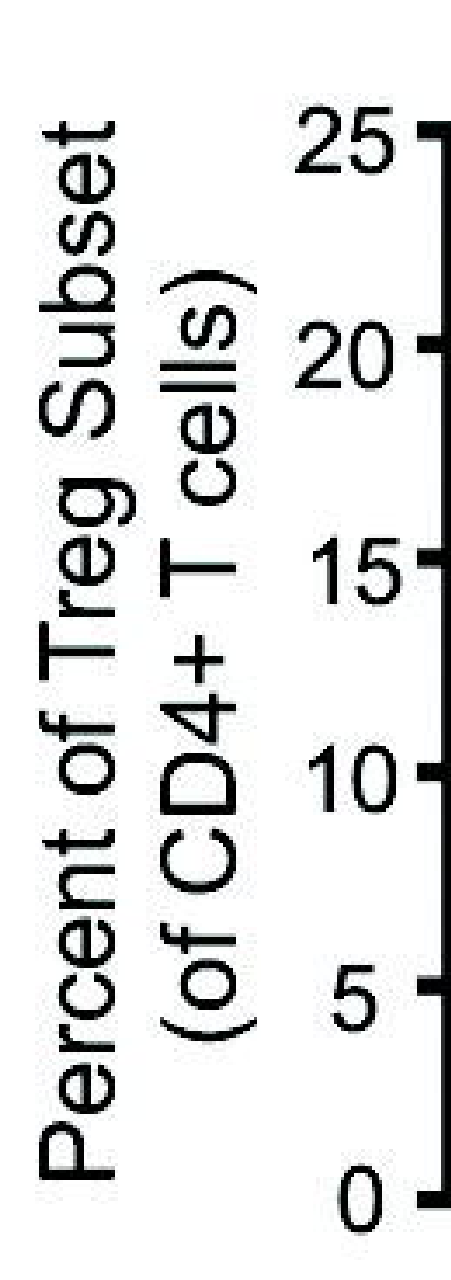

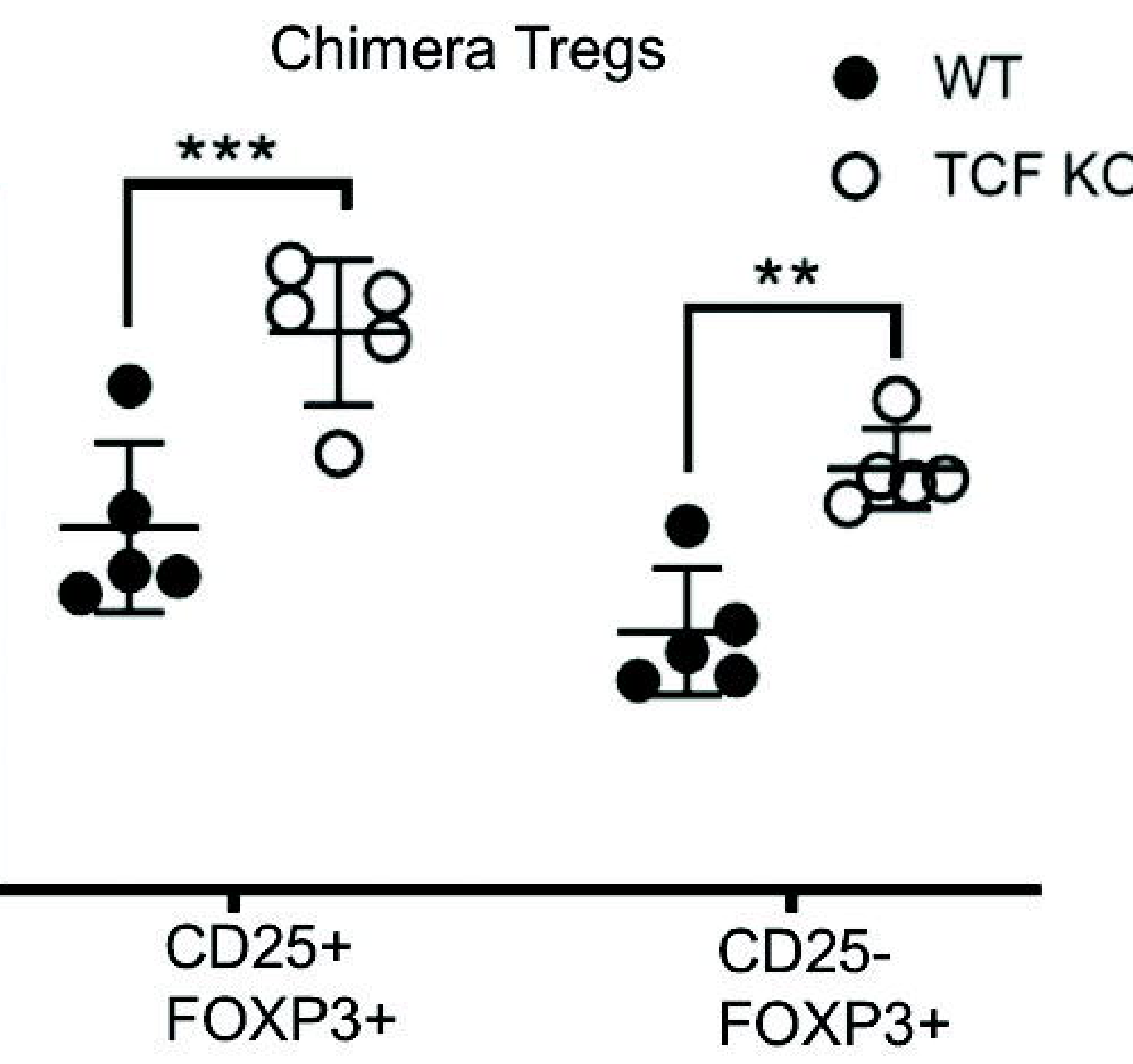




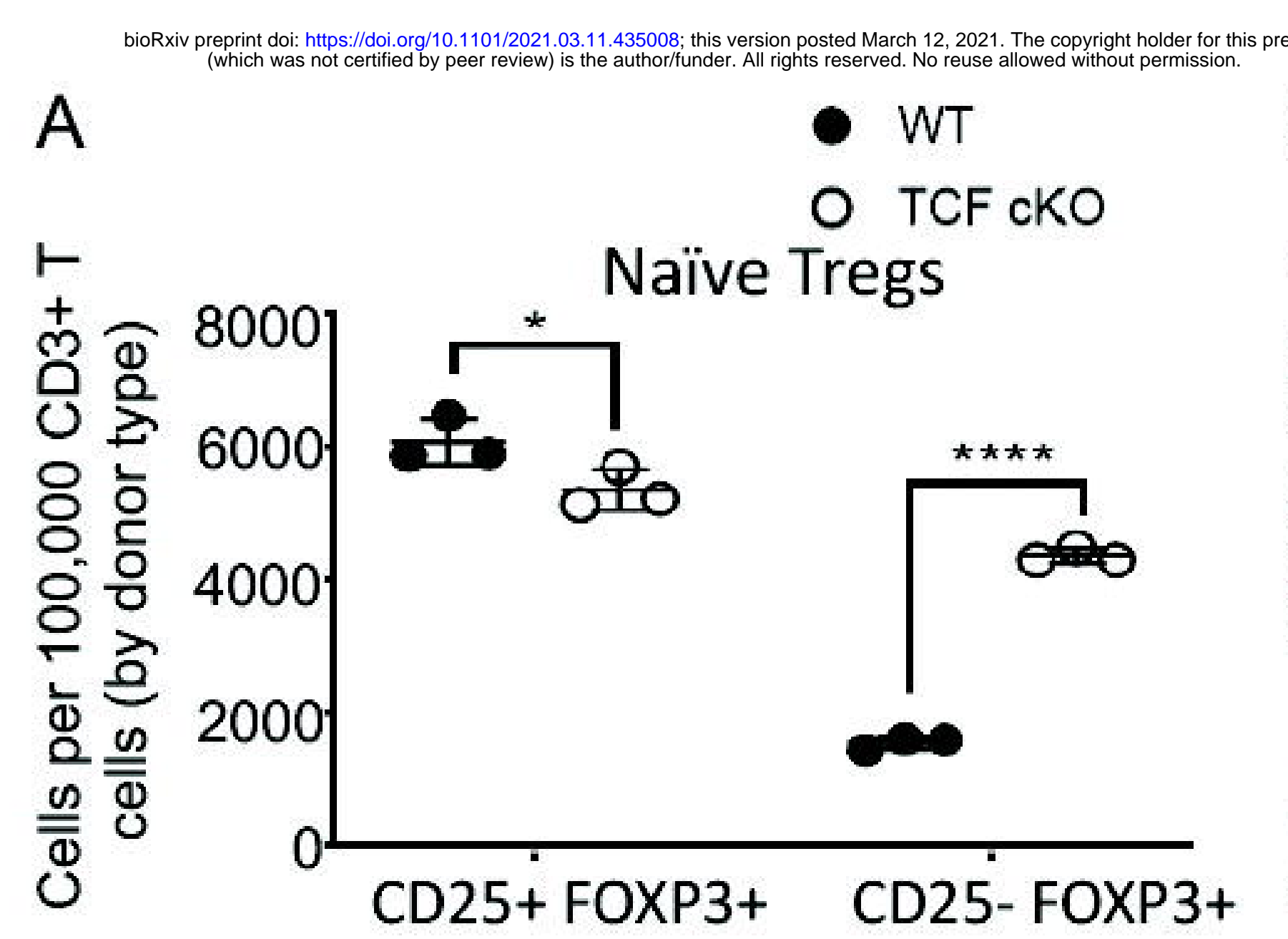

C

Chimera Tregs

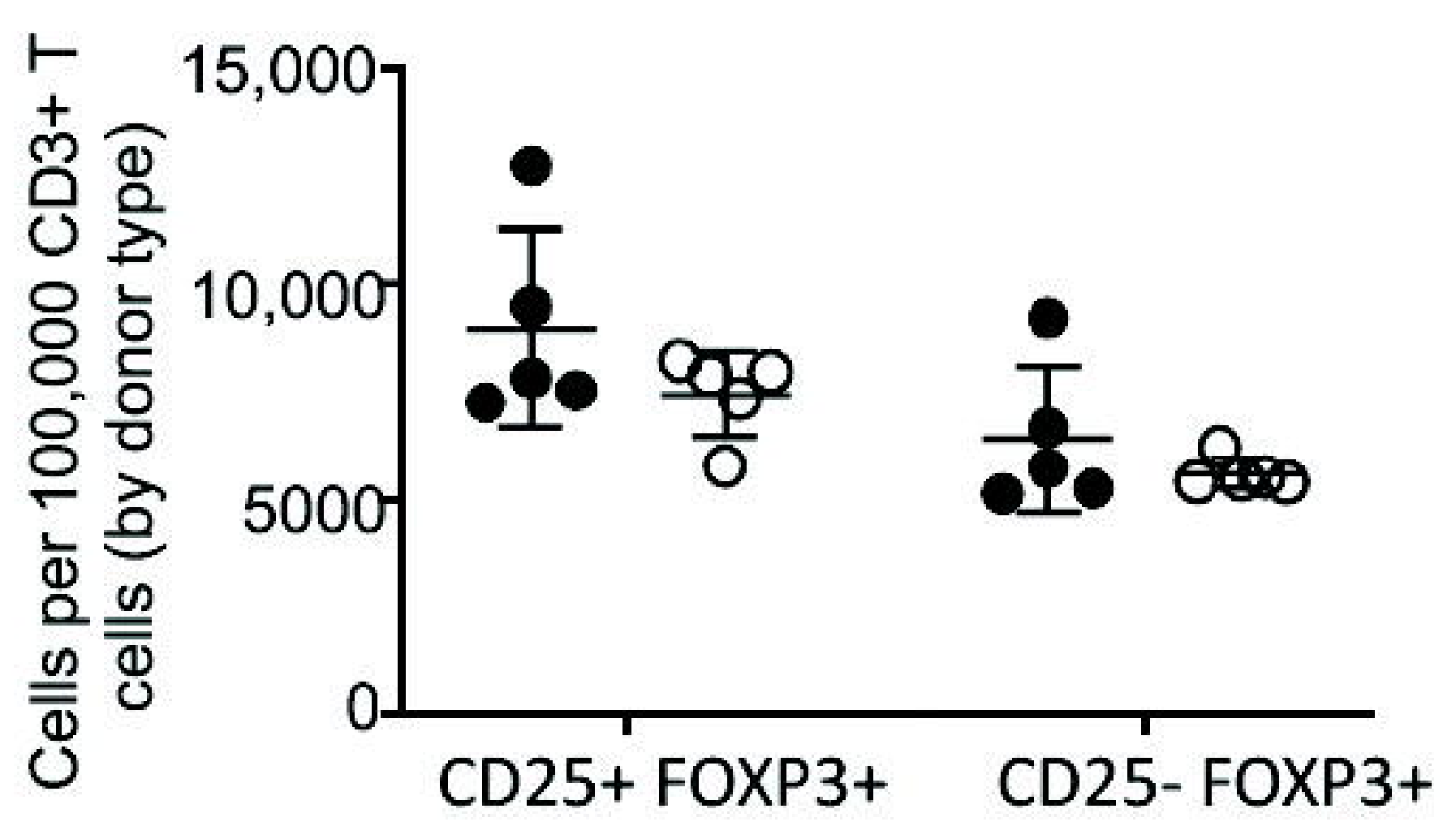

B

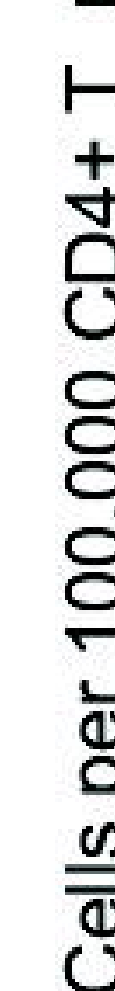

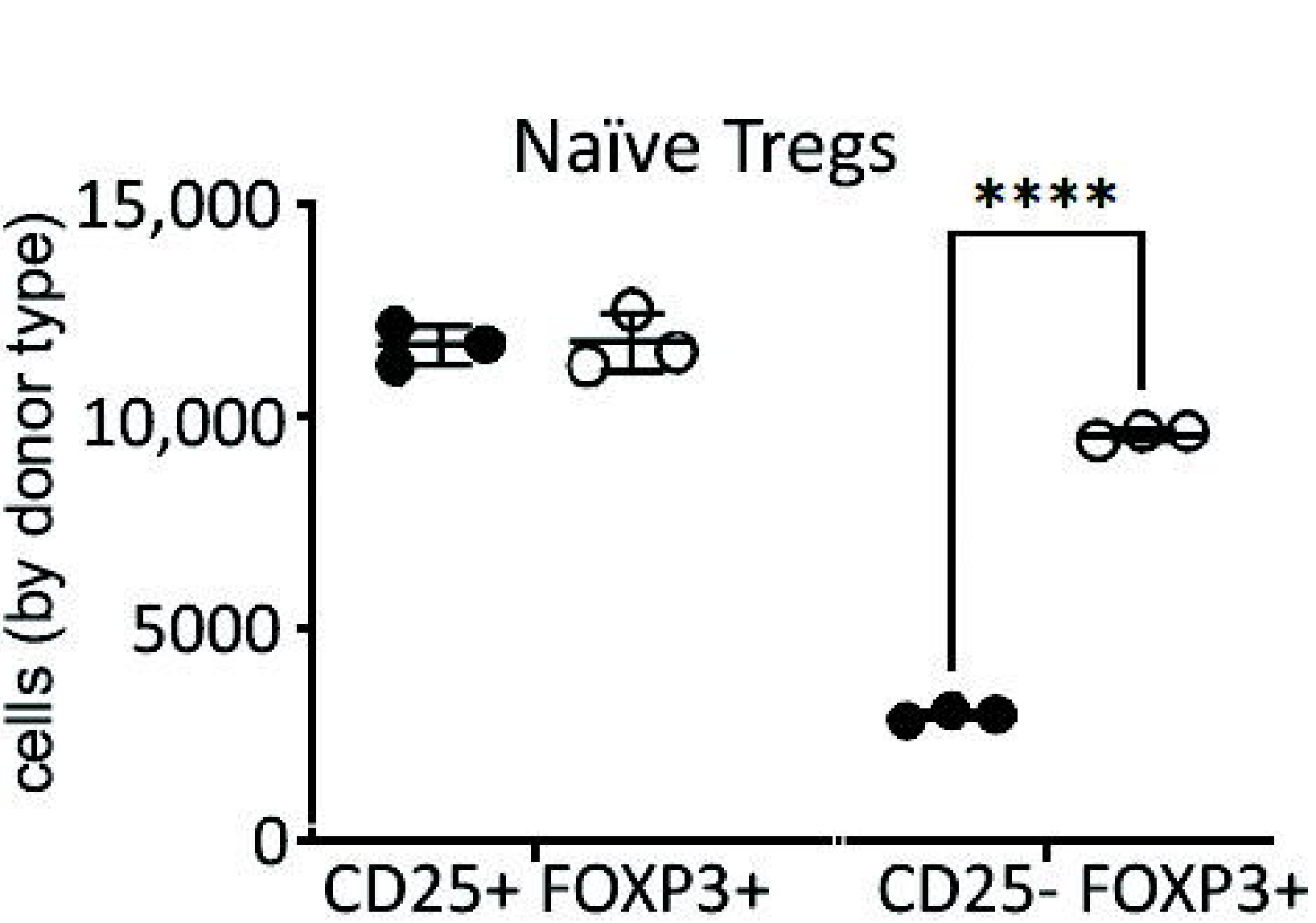

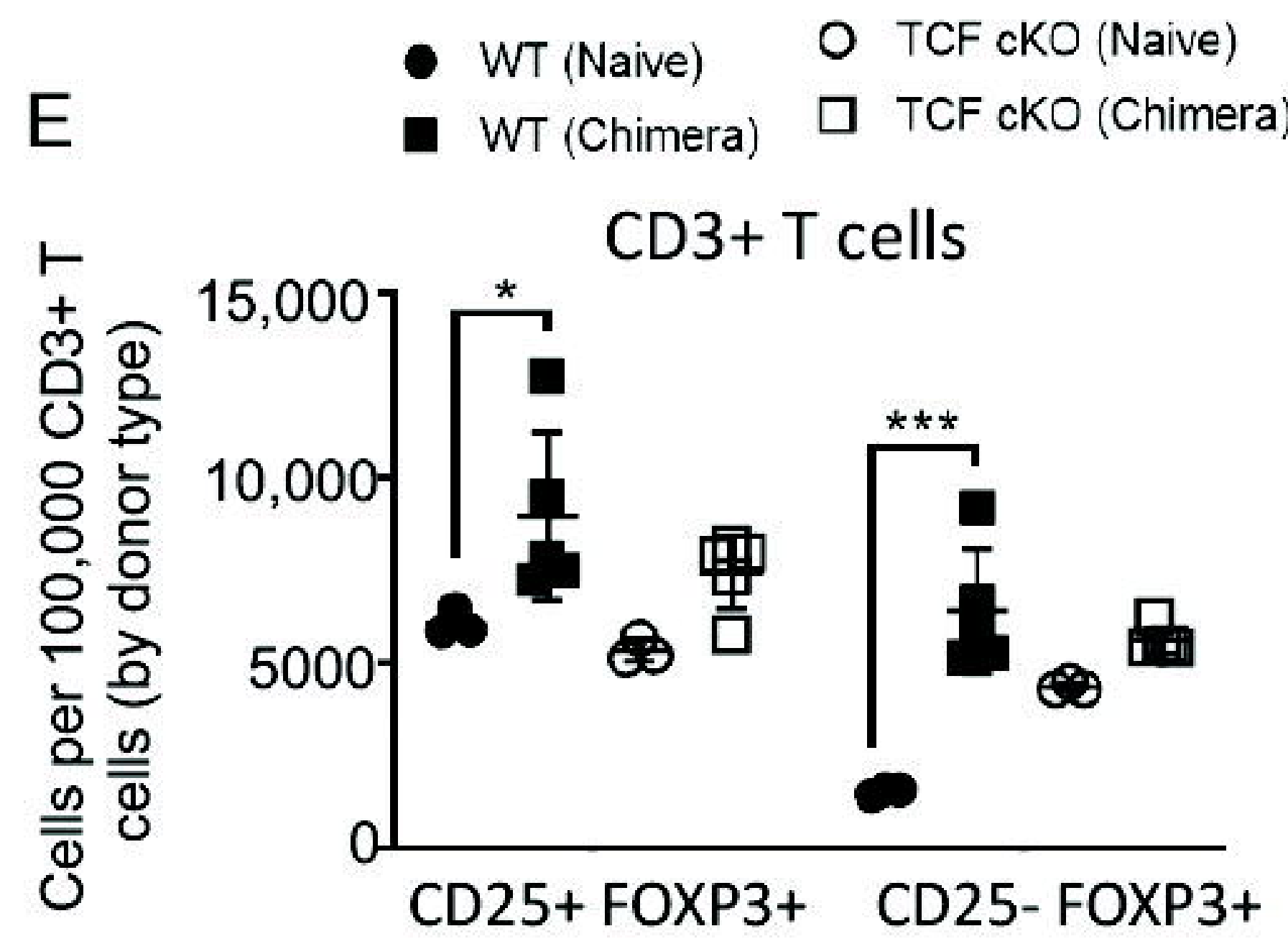

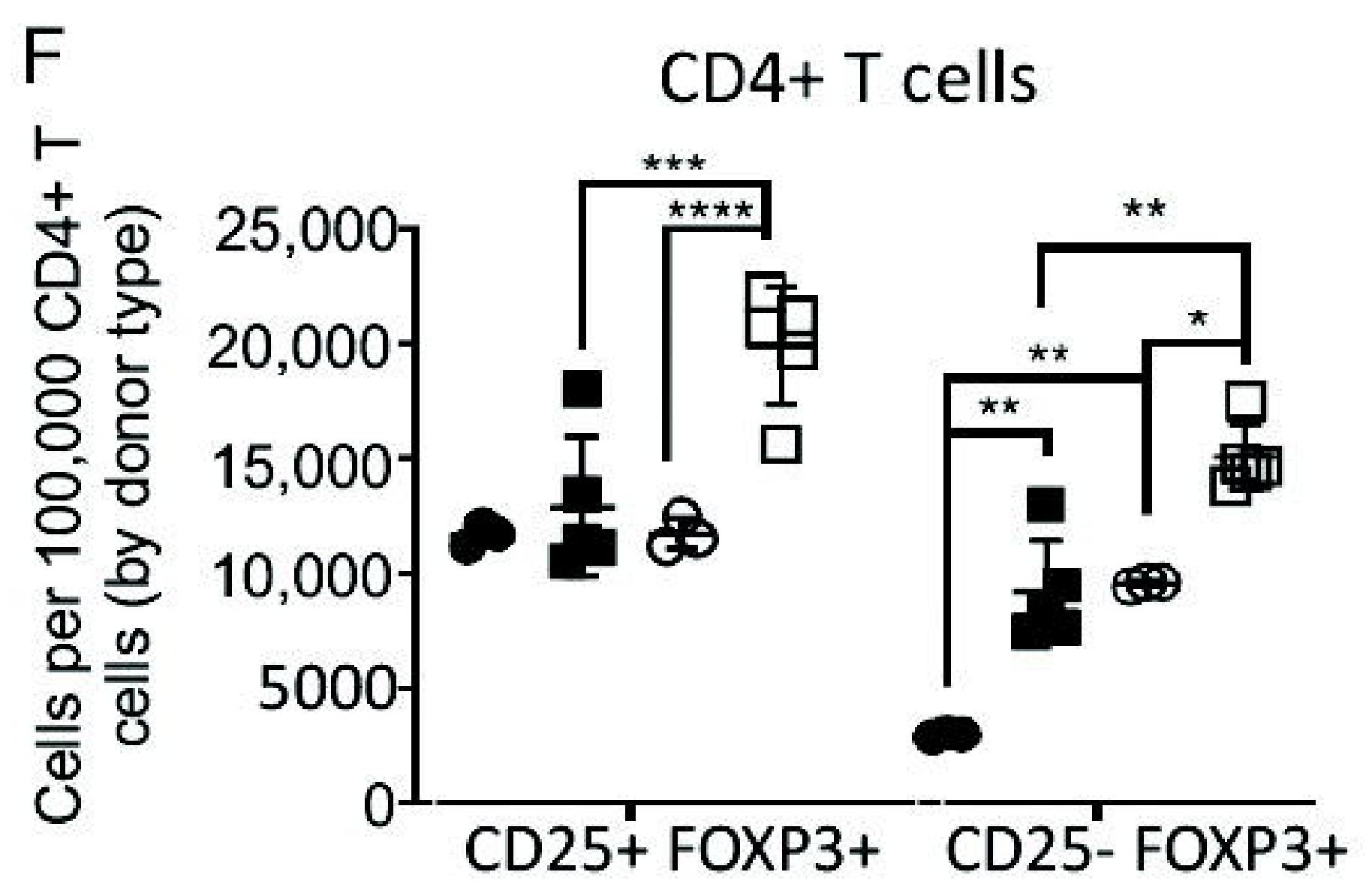


Fig 4

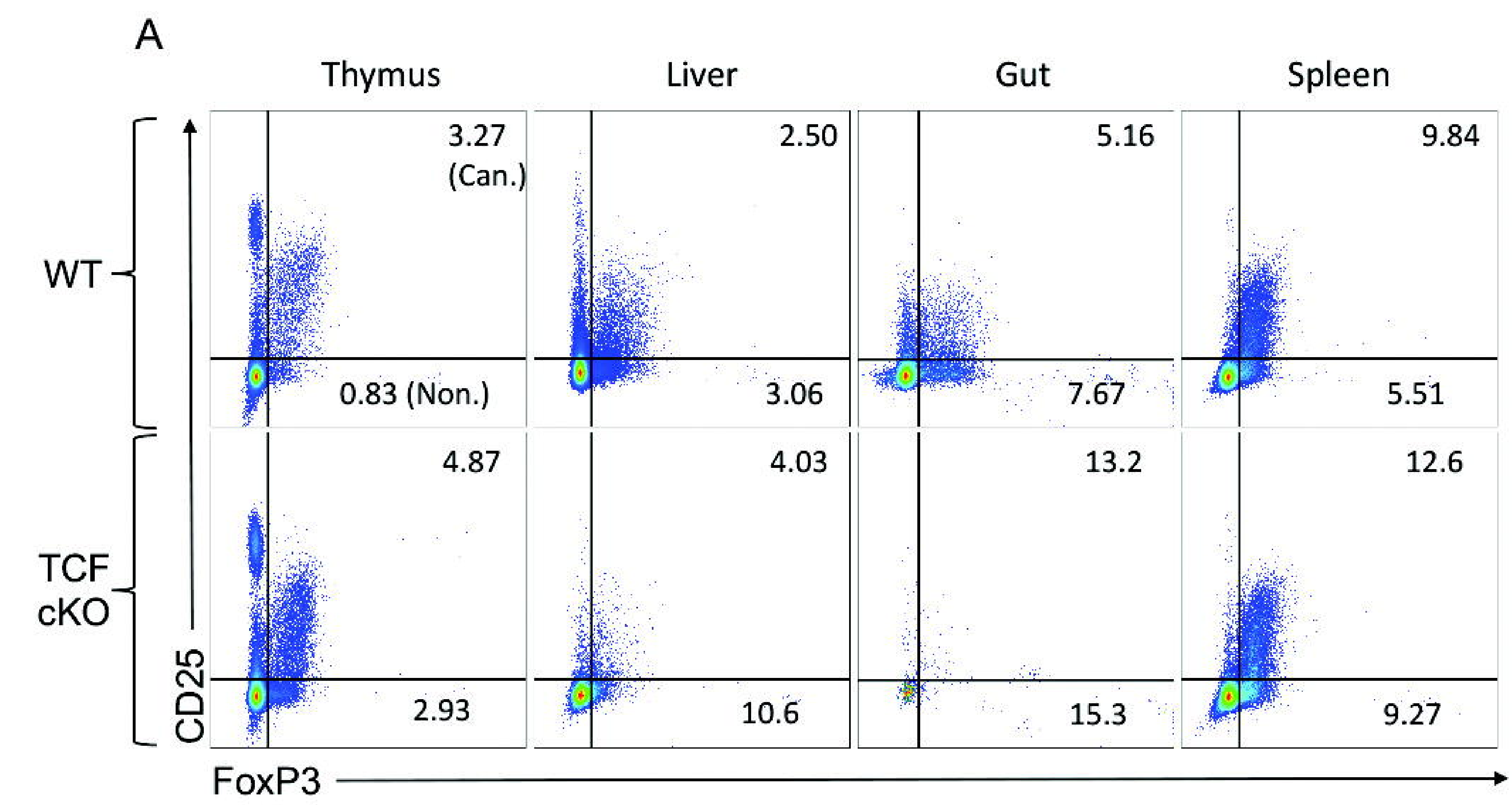

FoxP3
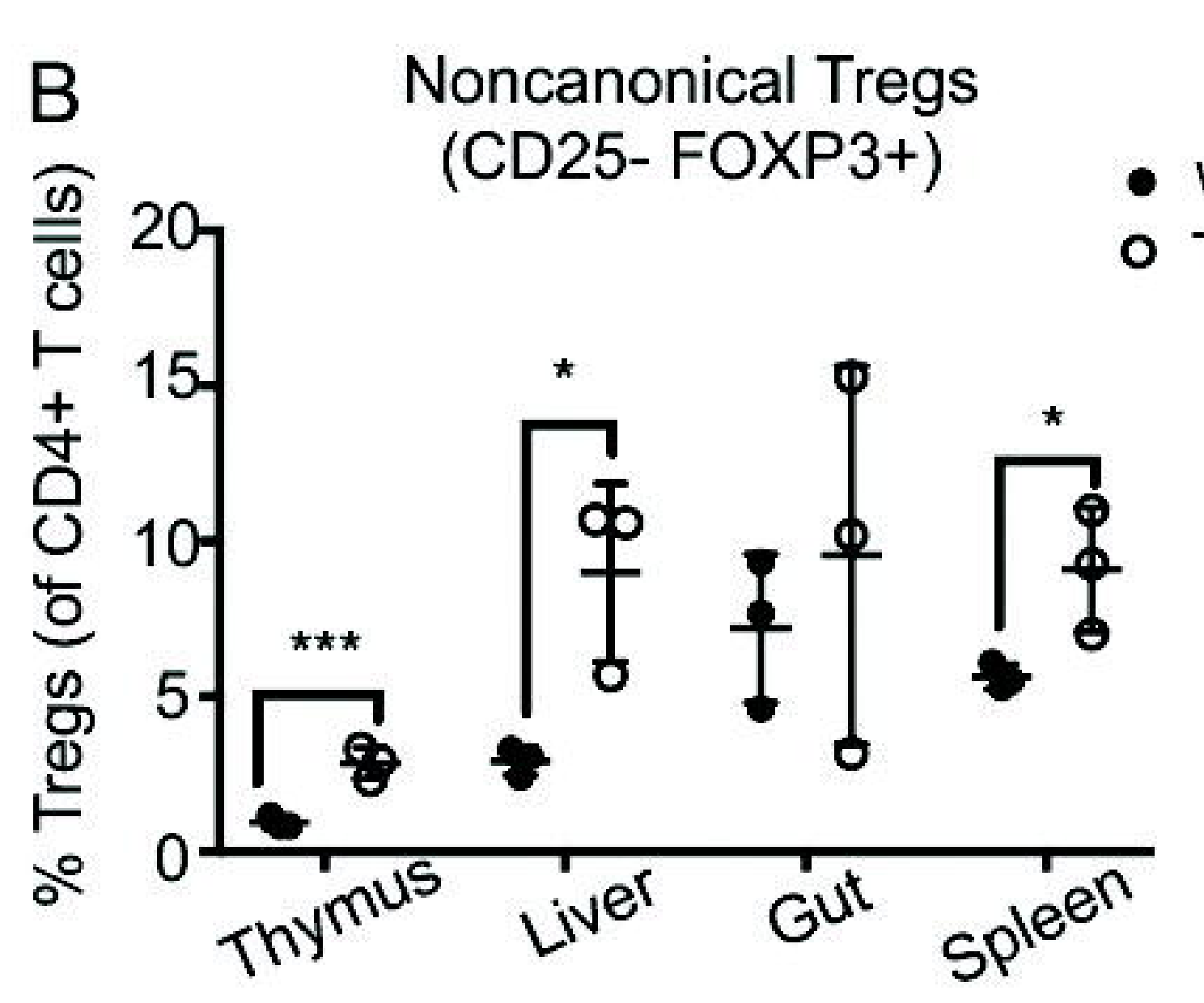

C

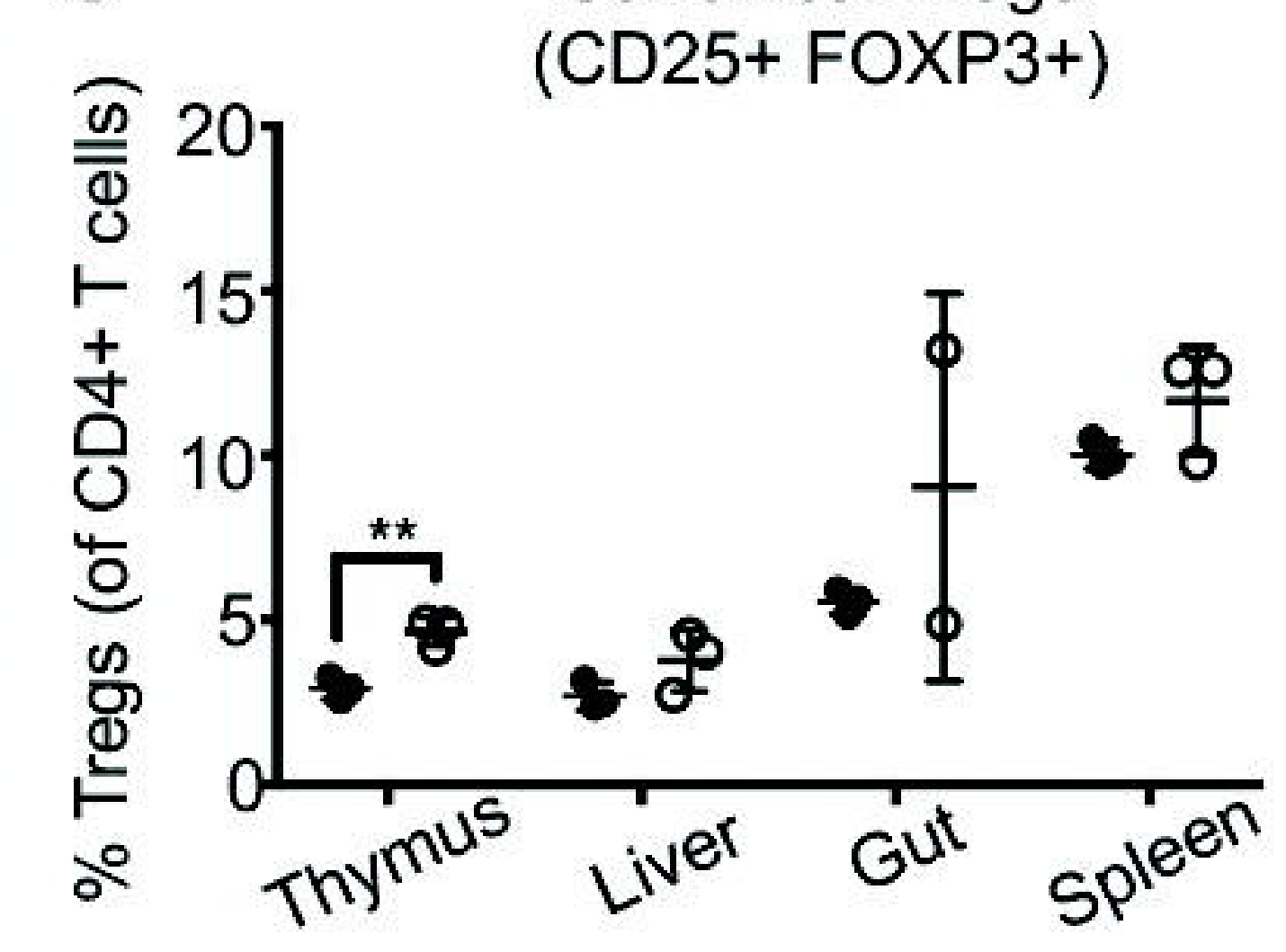

WT

- TCF KO

- TCF KO 


\section{Fig 5}
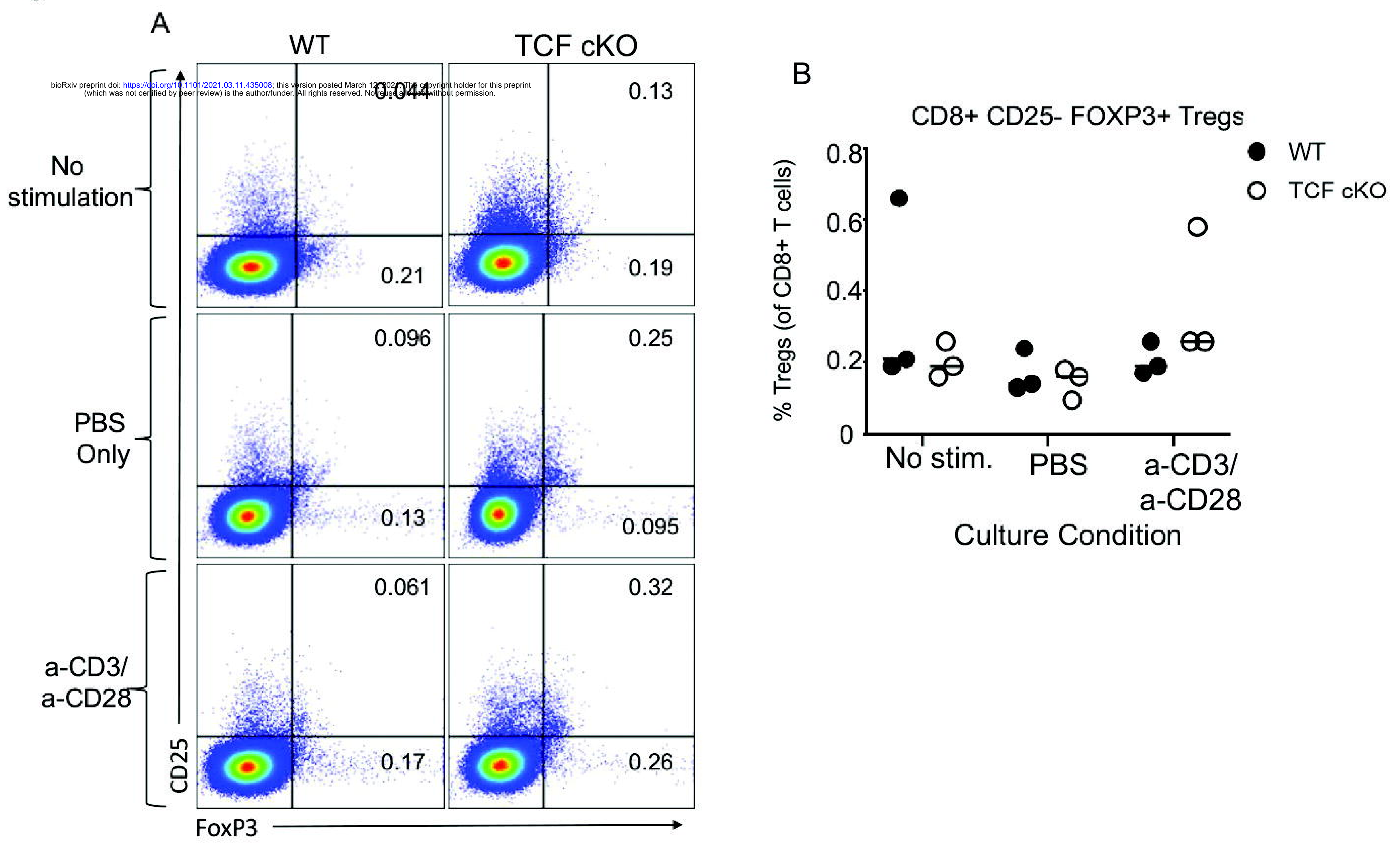


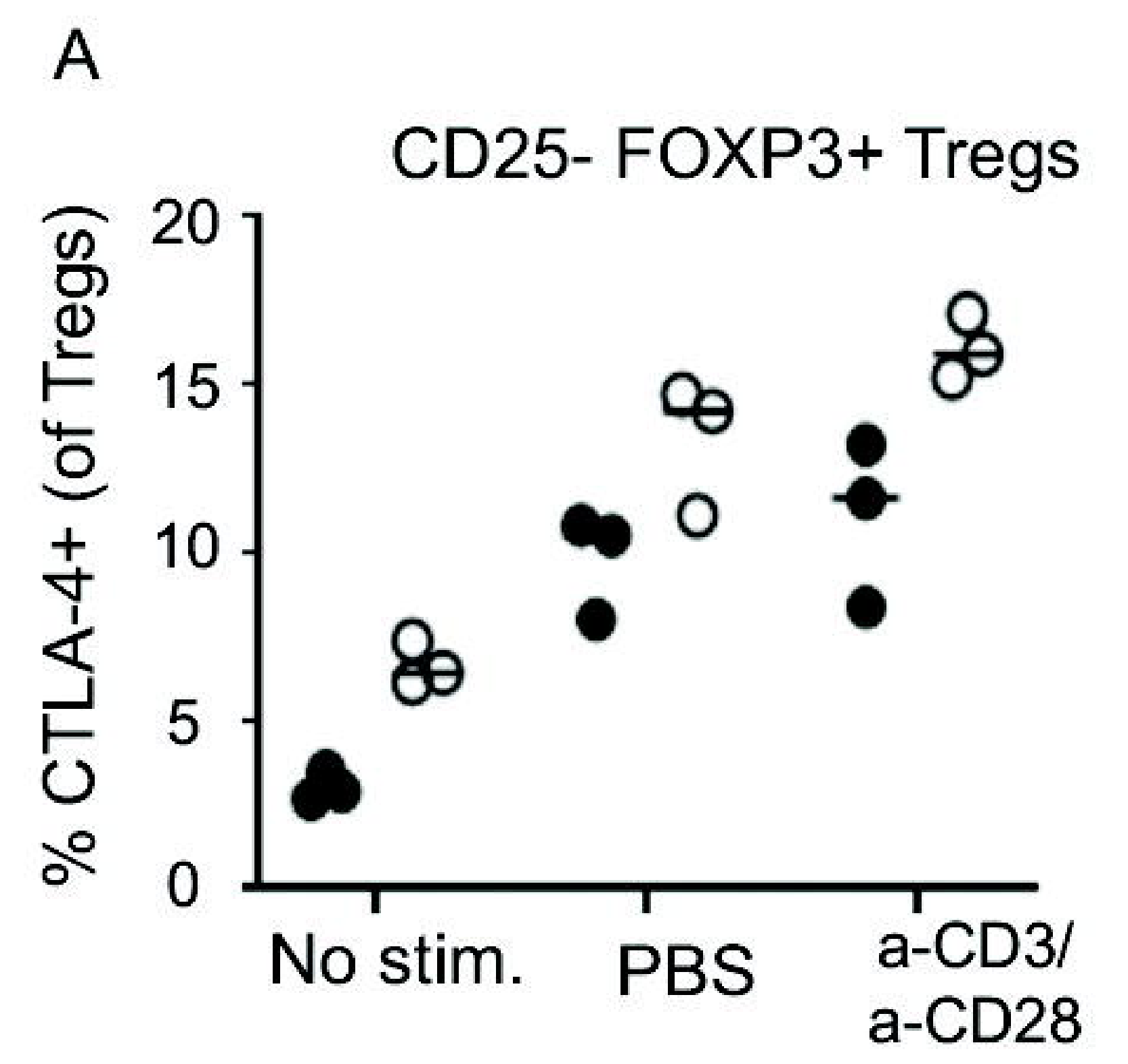

Culture Condition
B

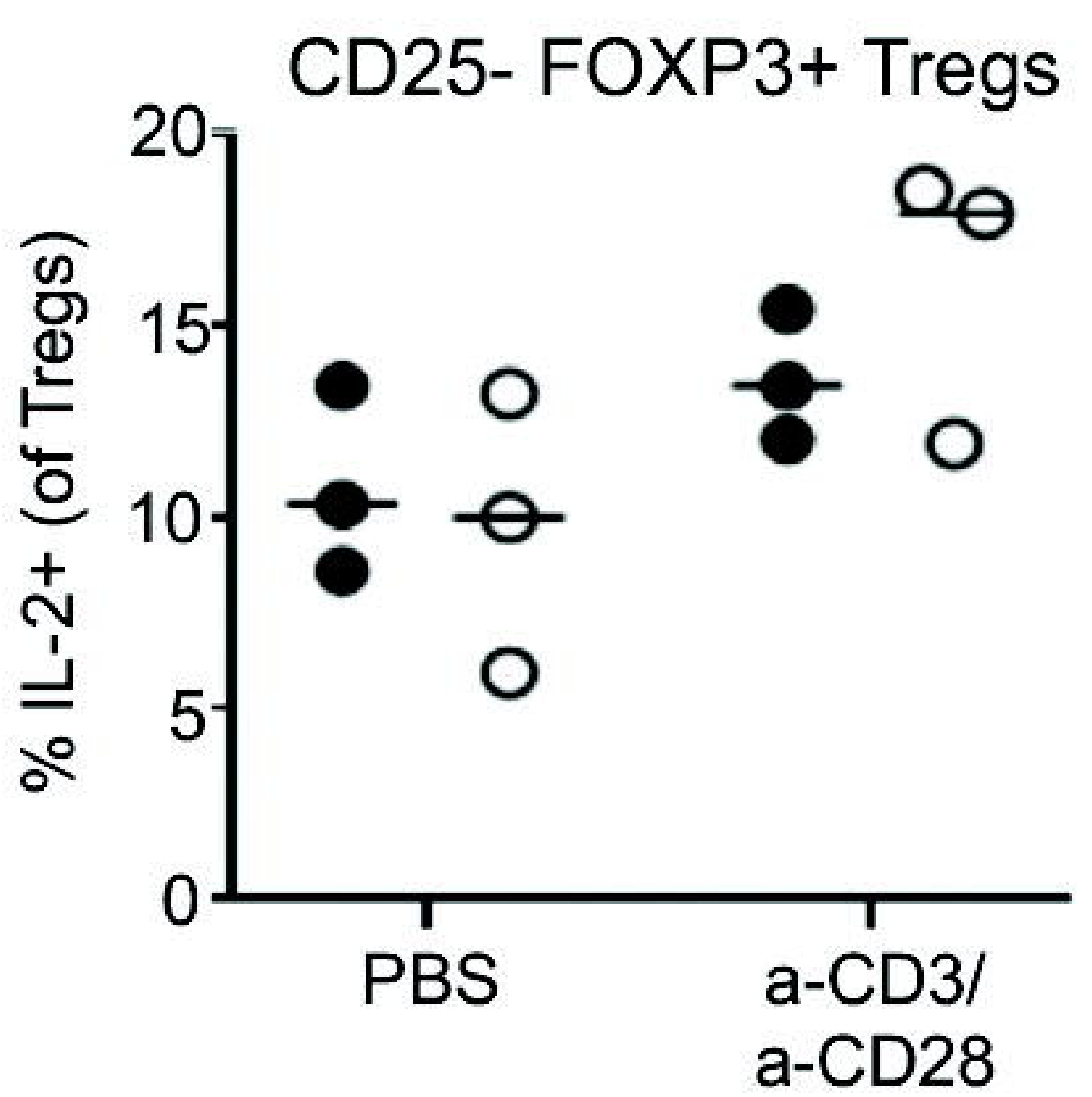

Culture Condition
C

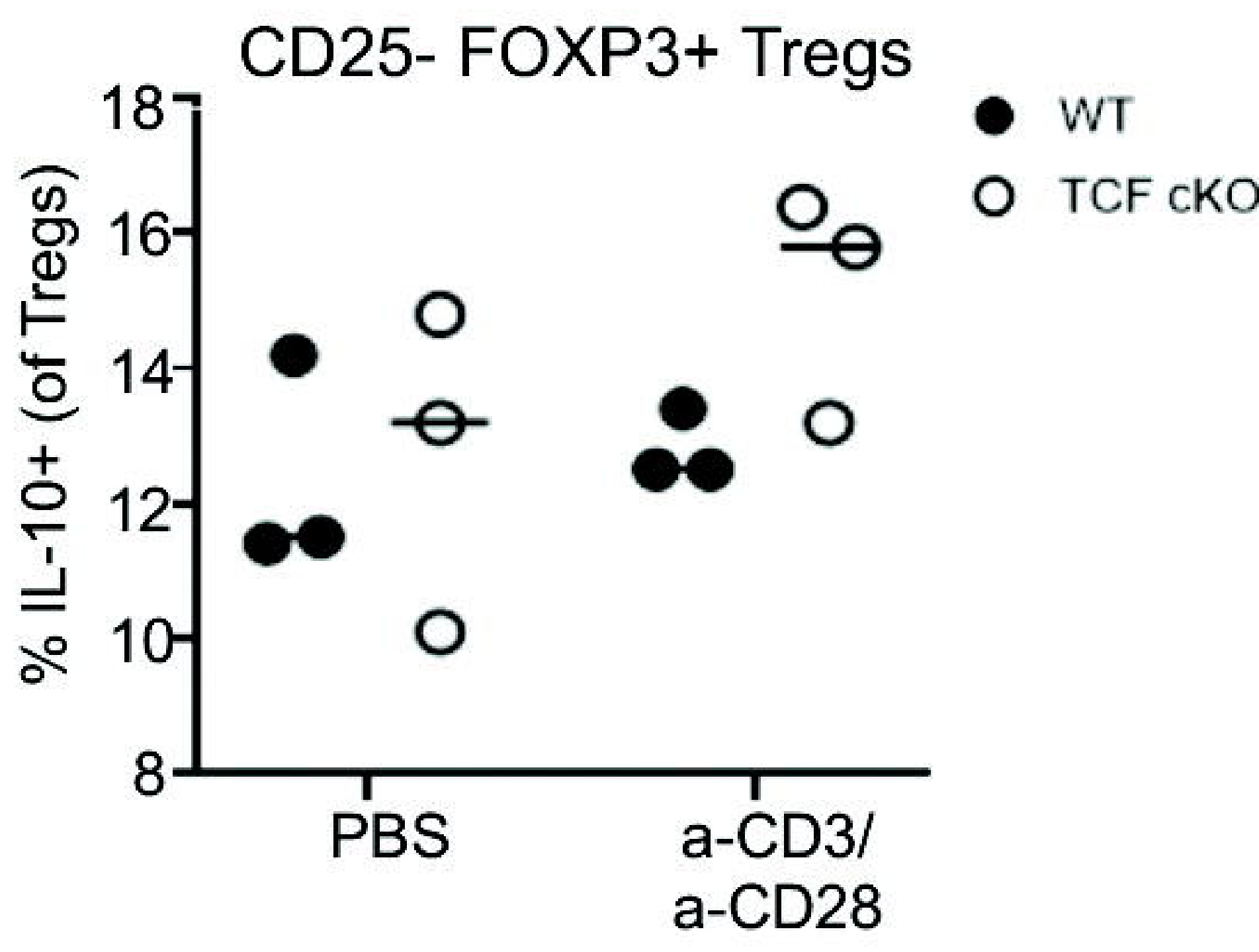

Culture Condition 